CUBO A Mathematical Journal

Vol.12, $N^{\underline{O}}$ 02, (235-259). June 2010

\title{
On Semisubmedian Functions and Weak Plurisubharmonicity
}

\author{
CHIA-CHI Tung ${ }^{1}$ \\ Dept. of Mathematics and Statistics, \\ Minnesota State University, Mankato, \\ Mankato, MN 56001, USA \\ email: chia.tung@mnsu.edu
}

\begin{abstract}
In this note subharmonic and plurisubharmonic functions on a complex space are studied intrinsically. For applications subharmonicity is characterized more effectually in terms of properties that need be verified only locally off a thin analytic subset; these include the submean-value inequalities, the spherical (respectively, solid) monotonicity, near as well as weak subharmonicity. Several results of Gunning [9, K and L] are extendable via regularity to complex spaces. In particular, plurisubharmonicity amounts (on a normal space) essentially to regularized weak plurisubharmonicity, and similarly for subharmonicity (on a general space). A generalized Hartogs' lemma and constancy criteria for certain matrix-valued mappings are given.
\end{abstract}

\section{RESUMEN}

En esta nota son estudiadas intrínsicamente las funciones subarmonicas y plurisubarmonicas sobre un espacio complejo. Para aplicaciones, subarmonicidad es caracterizada mas eficientemente en términos de propiedades que necesitan ser verificadas solamente localmente en un subconjunto analítico delgado; estas aplicaciones incluyen la desigualdad del valor-submedio, la monotonicidad esférica (respectivamente, sólida), bien como subarmonicidad debil. Varios resultados de Gunning $[9, \mathrm{~K}$ and $\mathrm{L}]$ son extendibles vía regularidad a espacios complejos. En particular, plurisubarmonicidad (sobre un espacio normal)

\footnotetext{
${ }^{1}$ Supports by the "Globale Methoden in der komplexen Geometrie" Grant of the German research society DFG and the Faculty Improvement Grant of Minnesota State University, Mankato, are gratefully acknowledged.
} 
importa esencialmente para plurisubarmonicidad débil regularizada y similarmente para subarmoniciada (sobre un espacio general). Son dados un lema de Hartogs generalizado y un criterio de constancia para ciertas aplicaciones matriz-valuada.

Key words and phrases: Subharmonicity, seminear subharmonicity, Jensen function, weak subharmonicity, weak plurisubharmonicity

2000 Math. Subj. Class.: Primary: 31C05; Secondary: 31C10, 32C15

\section{Introduction}

It is well-known that subharmonic functions are widely used in potential theory and partial differential equations, and their complex analogue, the plurisubharmonic functions, have played a significant role in the development of higher dimensional complex analysis. In this work such functions are studied on a general complex space from an intrinsic viewpoint, the main purpose here being to show that they admit intrinsic distributional representations despite the presence of singularities. In what follows let $Y$ denote a (reduced) complex space of pure dimension $m>0$. This means that $Y$ is a Hausdorff space which admits a countable basis of open sets and an open covering $\left\{U_{j}\right\}$ together with homeomorphisms $\alpha_{j}: U_{j} \rightarrow V_{j}$ where $V_{j}$ is a pure $m$-dimensional analytic subset in some open subset of $\mathbb{C}^{n_{j}}$ such that each mapping $\alpha_{j} \circ \alpha_{k}^{-1}: \alpha_{k}\left(U_{j} \cap V_{k}\right) \rightarrow \alpha_{j}\left(U_{j} \cap V_{k}\right)$ is biholomorphic. A Riemann covering of an $m$-dimensional complex space $Y$ at a point $a \in Y$ is a holomorphic map of a neighborhood of $a$ into $\mathbb{C}^{m}$ with discrete fibers. A mappping $\phi: Y \rightarrow[-\infty, \infty)$ is called subharmonic $(\phi \in S H(Y))$ if: (i) $\phi$ is upper semicontinuous $Y\left(\phi \in C^{\mathrm{usc}}(Y)\right)$; (ii) for any compact set $K \subset Y$ and any function $h: K \rightarrow \mathbb{R}$ which is continuous in $K$ and (locally) semiharmonic ([24]) in $\operatorname{int}(K)$, and $h(z) \geq \phi(z)$ for every $z \in \partial K$, it follows that $h(a) \geq \phi(a)$ for every $a \in K$ $([17$, p. 1$][10$, p. 16$])$. Subharmonicity is both a global and a local property (Lemma 3.2), the link between the two being rested with a maximum principle for almost everywhere solidly submedian functions (Proposition 3.1). In fact, via local Riemann coverings characterizations of subharmonicity can be given, as in the Euclidean case, in terms of the local property of being solidly, respectively, spherically, submedian. With the continuity assumption subharmonicity is also equivalent, by virtue of a maximum principle for seminearly subharmonic functions (Proposition 3.3), to near subharmonicity, spherical (respectively, solid) monotonicity. For applications it gains in usefulness if such a property needs only be verified locally off a thin analytic subset (Theorems 3.1 and 3.3). Especially, in the definition of subharmonicity, it suffices to require the condition (ii) to hold for closed pseudoballs $K$ lying in $Y$ off some thin analytic subset, instead of arbitrary compact subsets of $Y$ (by Lemma 3.1 and Theorem 3.1; cf. [1, p. 36]).

A locally integrable (or an upper semicontinuous) function $\psi: Y \rightarrow[-\infty, \infty)$ is said to be regularized at $a \in Y$ (respectively, regularized in $Y$ ) if

$$
\left.\nu_{p_{\Delta}}(a) \psi(a)=\lim _{\varepsilon \rightarrow 0}\langle\psi\rfloor \Delta\right\rangle_{a, \varepsilon}
$$

for some standard domain $\Delta$ at $a$ (respectively, the said condition holds for each $a \in Y$ ). An upper semicontinuous function $\psi: Y \rightarrow[-\infty, \infty)$ is said to be strictly regularized in $Y$, if $\psi$ satisfies not only the condition (1.1) for any standard domain $\Delta$ at every point of $Y$, but also the condition 


$$
\left.\nu_{p_{\Delta}}(a) \psi(a)=\lim _{\varepsilon \rightarrow 0}[\psi\rfloor \Delta\right]_{a, \varepsilon},
$$

for each pseudoball $\Delta$ at every point $a$ of $Y$. Here $\nu_{p}(a)$ denotes the multiplicity of a light holomorphic map $p$ at $a$ ([21, p. 22]; for a topological treatment see Radò and Reichelderfer [18]), and $\langle\psi\rfloor \Delta\rangle_{a, \varepsilon}$ (respectively, $\left.[\psi\rfloor \Delta\right]_{a, \varepsilon}$ ) the solid (respectively, spherical) mean-value function of $\psi((2.3)$ (2.4)). Every plurisubharmonic function is strictly regularized (Propositions 3.2 and 4.2).

A mapping $\phi: Y \rightarrow[-\infty, \infty)$ is plurisubharmonic $(\phi \in P S H(Y)$ in the sense of Lelong and Oka) iff $\phi \in C^{\text {usc }}(Y)$ and the pull-back of $\phi$ by any holomorphic map from the unit disc $\Delta \subset \mathbb{C}$ into $Y$ is subharmonic in $\Delta$. This definition turns out, owing to a notable result of Fornæss and Narasimhan $([5$, p. 64$])$, to be equivalent to one in an apparently stronger sense $([15$, p. 356]): a mapping $\phi$ : $Y \rightarrow[-\infty, \infty)$ is plurisubharmonic if $\phi$ has a plurisubharmonic extension into the ambient space of a local embedding of $Y$ at every point. Consequently plurisubharmonicity of a mapping is a local property. In contrast to "subharmonicity", such a mapping is necessarily radially submedian ((4.2)) and, if not identically equal to $-\infty$ on any open subset, a Jensen function (Proposition 4.2). On a normal complex space the (possibly weaker) property of being semiradially submedian suffices to characterize plurisubharmonicity (Theorem 4.1).

A locally integrable function with nonnegative local distributional Laplacian is called weakly subharmonic. With $C^{2}$-differentiability such a function can be regarded as possessing (off a thin analytic subset) nonnegative local spherical mean-radial derivative. By a similar token the requirement of semidefinite positivity of the distributional Levi form of locally integrable functions gives rise to a class $\mathcal{P} \mathcal{S H}_{\mathfrak{w}}(Y)$ of weakly plurisubharmonic functions. Denote by $S H_{\{\neq-\infty\}}(Y)$ the set of all subharmonic functions in $Y$ not identically equal to $-\infty$ on any open subset of $Y$. It turns out that a weakly subharmonic function $\phi$ belongs to $S H_{\{\neq-\infty\}}(Y)$ if and only if $\phi$ is regularized in $Y$ (Theorem 3.4). Owing to the strict regularity of plurisubharmonic functions, several results of Gunning [9, K and L] are extendable to complex spaces. Some of these are indicated in $\S 4$ and $\S 5$. Especially, given $\phi \in \mathcal{P} \mathcal{S H}_{\mathfrak{w}}(Y)$, there exists a unique locally integrable, regularized function $\psi: Y \rightarrow[-\infty, \infty)$ such that $\psi=\phi$ almost everywhere and $\psi \in S M_{\text {rad }}(Y) \cap P S H_{\{\neq-\infty\}}\left(Y_{\text {reg }}\right)$; if further $Y$ is normal, then a function $\phi: Y \rightarrow[-\infty, \infty)$ is weakly plurisubharmonic and regularized in $Y$ if and only if $\phi \in P S H_{\{\neq-\infty\}}(Y)$ (Theorem 4.2 and Corollary 4.1). As applications, a generalized Hartogs' lemma, conditions for the constancy of certain matrix-valued mappings (including extensions of two results of Bochner and Montgomery [2, p. 155]), and the maximum modulus principle for weakly real-analytic, subharmonic mappings, are given. Also, some lemmas and examples of recurring use on subharmonicity and plurisubharmonicity are gathered in an Appendix.

The author is indebted to the referee for suggestions which led to the improvement of this paper.

\section{Preliminaries}

Denote by $\|z\|$ the Euclidean norm of $z=\left(z_{1}, \cdots, z_{m}\right) \in \mathbb{C}^{m}$, where each component $z_{j}=x_{j}+i y_{j}$. Let the space $\mathbb{C}^{m}$ be oriented so that the Eulidean Kähler form $v^{m}:=\left((i / 2 \pi) \partial \bar{\partial}\|z\|^{2}\right)^{m}$ is positive. In what follows let $X, Y$ denote (reduced) complex spaces of pure dimension $m>0$, and let $p: Y \rightarrow \mathbb{C}^{m}$ be a holomorphic map. Set $a^{\prime}:=p(a), p^{[a]}:=p-a^{\prime}$, and $r_{a}:=\left\|p^{[a]}\right\|$ for each $a \in Y$. If $U \subseteq Y$ is an open set, $a \in U$ and $r>0$, set $U_{[a]}(r):=\left\{z \in U \mid r_{a}(z)<r\right\}$, and $U_{[a]}[r]:=\left\{z \in U \mid r_{a}(z) \leq r\right\}$. 
Denote by $\mathbb{B}_{\left[a^{\prime}\right]}(r)$ the open ball in $\mathbb{C}^{m}$ with center $a^{\prime}$ and radius $r$, omitting the subscript if $a^{\prime}=0$. Let $d v$ (respectively, $d \sigma_{r}$ ) be the Euclidean volume element of $\mathbb{C}^{m}$ (respectively, the sphere $\mathbb{S}(r)=\partial \mathbb{B}(r))$ and $|\mathbb{B}(r)|$ (respectively, $|\mathbb{S}|$ ) the volume of $\mathbb{B}(r)$ (respectively, $|\mathbb{S}(1)|$ ).

A complex space $X$ together with a holomorphic map $p: X \rightarrow \Omega$, where $\Omega$ is an open, connected subset of $\mathbb{C}^{m}$, is called a semi-Riemann domain (of dimension $m>0$ ) if there exists a thin analytic set $\Sigma$ in $\Omega$ such that $\Sigma_{p}:=p^{-1}(\Sigma)$ is thin in $X$, and the restriction $p: X^{0}:=X \backslash \Sigma_{p} \rightarrow \Omega_{0}:=\Omega \backslash \Sigma$ has discrete fibers; the map $p=\left(p_{1}, \cdots, p_{m}\right): X \rightarrow \Omega$ is called a Riemann semicovering. If $\Sigma=\emptyset$, then $(X, p)$ is called a Riemann domain and the map $p$ a Riemann covering. If $p: X \rightarrow \Omega$ is, in addition, a local homeomorphism, then $(X, p)$ is said to be unramified. Every proper modification of an affine algebraic variety is a semi-Riemann domain (as such it is parabolic in the sense of [22, pp. 73-74]). Riemann domains, in particular, analytic coverings of $\mathbb{C}^{m}$, play a fundamental role in complex analysis; in fact, every pure $m$-dimensional complex space is locally an analytic covering of a domain in $\mathbb{C}^{m}$ (hence a Riemann domain).

Let $(X, p)$ be a semi-Riemann domain; denote by $X^{*}$ the largest open subset of $X$ on which $p$ is locally biholomorphic. For each open subset $D \subseteq X$, set $D^{0}:=D \cap X^{0}$ and $D^{*}:=D \cap X^{*}$. Suppose that $p: \tilde{U} \rightarrow \Omega$, is a holomorphic map defined on an open neighborhood $\tilde{U}$ of a point $a \in Y$ such that: i) $p^{-1}\left(a^{\prime}\right) \cap \overline{\tilde{U}}=\{a\}$; ii) for a sufficiently small ball $U^{\prime}:=\mathbb{B}_{\left[a^{\prime}\right]}(r)$ in $\mathbb{C}^{m}, U_{a}:=$ $p^{-1}\left(U^{\prime}\right) \cap \tilde{U}=p^{-1}\left(U^{\prime}\right) \cap \overline{\tilde{U}}$ is connected and the mapping $\left.p\right\rfloor U_{a}: U_{a} \rightarrow U^{\prime}$ is an analytic covering (biholomorphic if $a \in D^{*}$ ); iii) every branch $V_{a}^{k}, k=1, \cdots, s_{a}$, of $U_{a}$ contains $a$; and iv)

$$
\left.s_{a}=\operatorname{deg}(p\rfloor U_{a}\right)=\nu_{p}^{y}(a)
$$

([21, Proposition 1.3]). Such an $U_{a}$ is called, for convenience, a pseudoball (of radius $r$ ) at a ([24, p. 557]). A pseudopolydisc $\Delta_{[a]}(R)$ over a polydisc (of polyradius $R$ ) in $\mathbb{C}^{m}$ is similarly defined. An open neighborhood $\Delta$ of a point $a$ in a complex space $Y$ is called a standard domain at $a$ if and only if there exists a Riemann covering $p: \Delta \rightarrow \Omega$ exhibiting $\Delta$ as either a pseudoball (of radius $R$ ) or a pseudopolydisc (of polyradius $R$ ) at $a$. If $p: Y \rightarrow \Omega$ is a Riemann semicovering and $a \in Y^{0}$, define $d(a)$ (respectively, $\left.d_{\mathrm{pd}}(a)\right):=$ the supremum of $R>0$ (respectively, $R=\left(R_{1}, \cdots, R_{m}\right)>(0, \cdots, 0)$ ) such that a pseudoball (respectively, polydisc) $\Delta_{[a]}(R)$ exists.

The notions of $C^{k}$-differential forms, the exterior differentiation $d$, the operators $\partial, \bar{\partial}$ and $d^{c}:=$ $(1 / 4 \pi i)(\partial-\bar{\partial})$, are well-defined on a complex space $Y$ despite the presence of singularities ([23, Chapter 4]). If $G \subseteq Y$ is open subset, denote by $d G$ the (maximal) boundary manifold of $G_{\text {reg }}$ in $Y_{\text {reg }}$, the manifold of simple points of $Y$, oriented to the exterior of $G_{\text {reg }}\left(\left[23\right.\right.$, p. 218]). If $p: G \rightarrow \mathbb{C}^{m}$ is a holomorphic map and $a \in G$, the form

$$
v_{p}:=d d^{c} r_{a}^{2}=\frac{i}{2 \pi} \partial \bar{\partial} r_{a}^{2}
$$

is nonnegative $([23, \S 4])$ and independent of $a$. The Poincaré form

$$
\sigma_{a}:=\frac{2 d^{c} r_{a}}{r_{a}^{2 m-1}} \wedge v_{p}^{m-1}
$$

is $d$-closed. Also set $d \tilde{v}:=p^{*} d v, d \sigma_{[a], r}:=\left(p^{[a]}\right)^{*} d \sigma_{r}$, where $d v$ (respectively, $d \sigma_{r}$ ) denotes the Euclidean volume element of $\mathbb{C}^{m}$ (respectively, $\mathbb{S}(r)$ ). If $p: Y \rightarrow \mathbb{C}^{m}$ is a Riemann semicovering and $\phi \in C^{\text {usc }}(U)$, where $U=U_{[a]}\left(r_{0}\right) \subset Y$, the associated spherical mean-value function is defined by 


$$
[\phi\rfloor U]_{a, r}:=\int_{d U_{[a]}(r)} \phi \sigma_{a}, \quad 0<r<r_{0},
$$

([24, (3.2)]). Similarly, if $\phi \in C^{\text {usc }}(\Delta)$, where $\Delta=\Delta_{[a]}\left(R_{0}\right)$ is a standard domain in $Y$, the associated solid mean-value function is defined by

$$
\langle\phi\rfloor \Delta\rangle_{a, R}:=\frac{1}{\operatorname{vol}\left(\Delta\left(a^{\prime}, R\right)\right)} \int_{\Delta_{a}(R)} \phi(z) d \tilde{v}(z), \quad 0<R<R_{0},
$$

where $\operatorname{vol}\left(\Delta\left(a^{\prime}, R\right)\right)$ denotes the Euclidean volume of the induced domain $\Delta\left(a^{\prime}, R\right):=p\left(\Delta_{a}(R)\right)$. Let $\mathrm{j}_{d G}: d G \rightarrow Y$ denote the inclusion mapping and $d \sigma=d \sigma_{d G}$ the (Lebesgue) surface measure on $Y^{*} \cap d G$ induced by the local patches $p_{U}:=p: U \rightarrow \mathbb{B}_{\left[a^{\prime}\right]}(r)$ on an unramified neighborhood $U$ of a point $a \in d G$.

\section{Subharmonicity}

An upper semicontinuous map $\phi: Y \rightarrow[-\infty, \infty)$ is called (locally) semispherically submedian in $Y$ $\left(\phi \in S M_{\mathrm{sm} . \mathrm{sph}}(Y)\right)$, if there exists a thin analytic subset $A$ of $Y$ such that: (i) at every $z \in Y \backslash A$, there is a pseudoball $\Delta$ of radius $r_{0}$ for which the inequality

$$
\left.\nu_{p_{\Delta}}(z) \phi(z) \leq[\phi\rfloor \Delta\right]_{z, r}, \quad 0<r<r_{0},
$$

holds; (ii) if $\phi$ admits an exceptional peak point, that is, a point $z_{*} \in A$ with $\phi\left(z_{*}\right)=\sup _{Y} \phi$, then $\phi\left(z_{*}\right) \leq \liminf _{n \rightarrow \infty} \phi\left(a_{n}\right)$ for all sequences $\left\{a_{n}\right\}$ in $\Delta \backslash A$ converging to $z_{*}$. Similarly, the set $S M_{\text {sm.sol }}(Y)$ of (locally) semisolidly submedian maps is defined by requiring (in place of (3.1)) the inequality

$$
\left.\nu_{p_{\Delta}}(z) \phi(z) \leq\langle\phi\rfloor \Delta\right\rangle_{z, R}, \quad 0<R<R_{0},
$$

to hold for some pseudoball $\Delta=\Delta_{z}\left(R_{0}\right)$. If in the preceding the set $A$ can be taken to be the empty set, then $\phi$ is called spherically submedian (respectively, solidly submedian) in $Y$.

An upper semicontinuous map $\phi: Y \rightarrow[-\infty, \infty)$ is said to be weakly Jensen if there exists a thin analytic subset $A$ of $Y$ such that at each $z \in Y_{0}(\phi):=Y \backslash A$, the inequality (3.2) holds with respect to some standard domain $\Delta$, where, in the case of a pseudoball (respectively, pseudopolydisc), $R_{0}$ denotes the radius (respectively, the polyradius) of $\Delta$. (In view of Proposition 4.1 and Theorem 3.1) the following extension of [24, Proposition 3.2] generalizes the maximum principle for subharmonic (and plurisubharmonic) functions:

Proposition 3.1. (Maximum principle for weakly Jensen functions) Let $D$ be a domain in $Y$ and $\phi: D \rightarrow[-\infty, \infty)$ a weakly Jensen function with finite supremum $M$. Assume that (i) the peak set $\mathcal{P}:=\{z \in D \mid \phi(z)=M\}$ is nonempty; (ii) if no peak point of $\phi$ lies in $D_{0}:=D_{0}(\phi)$, then every sequence $\left\{a_{n}\right\}$ in $D_{0}$ converging to a point $z_{0} \in \mathcal{P}$ satisfies the inequality $\phi\left(z_{0}\right) \leq \liminf _{n \rightarrow \infty} \phi\left(a_{n}\right)$. Then $\phi=$ constant. 
Proof. Let $F:=\{z \in D \mid \phi(z)<M\}$. Assume at first that $\phi$ admits a peak point in $D_{0}$. Choose a standard domain $\Delta \subseteq D_{0}$ at such a point $a$ for which the inequality (3.2) holds. Then the proof of [24, Proposition 3.2] carries over here and yields the desired conclusion. Now consider the case where $\phi$ has no peak point in $D_{0}$. Let $z_{0}$ be any peak point of $\phi$, and $\left\{a_{n}\right\}$ a sequence in $D_{0}$ converging to $z_{0}$. Choose a sequence of standard domains $\Delta_{\left[a_{n}\right]}\left(R_{n}\right)$ (relative to a Riemann covering $p_{n}$ ) with norm $\left\|R_{n}\right\| \rightarrow 0$ as $n \rightarrow \infty$ such that the solid submean-value property (3.2) holds. Suppose that $F \cap B_{\left[z_{0}\right]}\left(R_{k}\right) \neq \emptyset$ for every standard domain $B_{\left[z_{0}\right]}\left(R_{k}\right)$. Then $\phi(z)<M$ for all $z$ in a neighborhood of each point in $F \cap B_{\left[z_{0}\right]}\left(R_{k}\right)$. Since $\Delta_{\left[a_{n}\right]}\left(R_{n}\right) \cap B_{\left[z_{0}\right]}\left(R_{k}\right) \neq \emptyset$ for sufficiently large $n$ and $a_{n} \in F$, it follows from [23, Proposition 5.2.2] and $[24,(2.4)]$ that

$$
\begin{aligned}
\operatorname{vol}\left(\Delta\left(a_{n}^{\prime}, R_{n}\right)\right) \nu_{p_{n}}\left(a_{n}\right) \phi\left(a_{n}\right) & \leq \int_{\Delta_{a_{n}}\left(R_{n}\right)} \phi(z) d \tilde{v}(z) \\
& \left.<\int_{\Delta_{a_{n}}\left(R_{n}\right)} M d \tilde{v}(z)=M \operatorname{vol}\left(\Delta\left(a_{n}^{\prime}, R_{n}\right)\right) \operatorname{deg}\left(p_{n}\right\rfloor \Delta\right) .
\end{aligned}
$$

This inequality and the relation $[24,(2.5)]$ imply that

$$
\phi\left(z_{0}\right) \leq \liminf _{n \rightarrow \infty} \phi\left(a_{n}\right)<M,
$$

hence a contradiction. Therefore $F \cap B_{\left[z_{0}\right]}\left(R_{k}\right)=\emptyset$ for some $B_{\left[z_{0}\right]}\left(R_{k}\right)$. Thus the set $\mathcal{P}$ is open and nonvoid. Then by the connectedness of $D$ one must have $F=\emptyset$. Consequently $\phi(z) \equiv M$ in $D$.

In the following, if $V^{k}$ is a branch of a standard domain $\Delta$ at point $a \in Y$, and $\phi \in L^{1}(\Delta)$, denote by $\tilde{\phi}_{k}$ a locally integrable function on $\Delta^{\prime}$ such that $p^{*} \tilde{\phi}_{k}=\phi$ on $V^{k}$. Since each branch $V^{k}$ contains $a$, the function $\tilde{\phi}_{k}$ can be chosen to be continuous in $\Delta^{\prime}$, provided so is $\phi$ in $\Delta$.

Lemma 3.1. Let $(X, p)$ be a Riemann domain. Assume that $\phi: X \rightarrow[-\infty, \infty)$ is upper semicontinuous, and for each pseudoball $U \subset X$ and every continuous function $h: \bar{U} \rightarrow \mathbb{R}$ with $h \geq \phi\rfloor \partial U$ such that $h$ is semiharmonic in $U$, one has $h \geq \phi\rfloor U$. Then $\nu_{p}(a) \phi(a) \leq[\phi]_{a, r}$ for all $a \in X$ and all $r \in(0, d(a))$, (in particular, $\phi$ is spherically submedian).

Proof. Let $U$ be a pseudoball at $a \in X$, and $r \in(0, d(a))$. There exists, for each branch $V^{k}, k=$ $1, \cdots, l$, of $U$, a decreasing sequence of continuous functions $\tilde{\phi}_{k}^{n}$ on $U_{a^{\prime}}^{\prime}[r]$ converging pointwise to $\tilde{\phi}_{k}$. The Poisson integral $h_{n}:=P_{a^{\prime}, r}\left(\tilde{\phi}_{k}^{n}\right)$ is continuous and harmonic in $U_{a^{\prime}}^{\prime}(r)$, and $h_{n}=\tilde{\phi}_{k}^{n} \geq \tilde{\phi}_{k}$ on $\partial U_{a^{\prime}}^{\prime}(r)$. By the subharmonicity of $\tilde{\phi}_{k}$ and the formula [25, (4.16)], one has

$$
\tilde{\phi}_{k}\left(z^{\prime}\right) \leq h_{n}\left(z^{\prime}\right)=\int_{\partial U_{\left[a^{\prime}\right]}^{\prime}(r)} \mathcal{P}_{a^{\prime}, r}\left(z^{\prime}, \zeta^{\prime}\right) \tilde{\phi}_{k}^{n}\left(\zeta^{\prime}\right) d \sigma_{\left[a^{\prime}\right], r},
$$

for each $z^{\prime} \in U_{a^{\prime}}^{\prime}(r)$. Hence by the monotone convergence theorem,

$$
\tilde{\phi}_{k}\left(z^{\prime}\right) \leq \lim _{n \rightarrow \infty} h_{n}\left(z^{\prime}\right)=\int_{\partial U_{\left[a^{\prime}\right]}^{\prime}(r)} \mathcal{P}_{a^{\prime}, r}\left(z^{\prime}, \zeta^{\prime}\right) \tilde{\phi}_{k}\left(\zeta^{\prime}\right) d \sigma_{\left[a^{\prime}\right], R}
$$

In particular, by the definition (2.3), 


$$
\tilde{\phi}_{k}\left(a^{\prime}\right) \leq\left[\tilde{\phi}_{k}\right]_{a^{\prime}, r}=\int_{\partial U_{\left[a^{\prime}\right]}^{\prime}(r)} \frac{1}{r|\mathbb{S}|} \frac{r^{2}}{\left\|a^{\prime}-\zeta^{\prime}\right\|^{2 m}} \tilde{\phi}_{k}\left(\zeta^{\prime}\right) d \sigma_{\left[a^{\prime}\right], r} .
$$

Consequently $\phi$ satisfies the spherical submean-value inequality (3.1) for all $r \in(0, d(a))$.

Observe that for each $\psi \in L^{1}\left(\mathbb{B}_{\left[a^{\prime}\right]}\left[r_{0}\right]\right)$, the Fubini type formula holds:

$$
\int_{\mathbb{B}_{\left[a^{\prime}\right]}(r)} \psi d v_{\left[a^{\prime}\right]}=\int_{0}^{r}\left(\int_{\mathbb{S}_{\left[a^{\prime}\right]}(t)} \psi d \sigma_{\left[a^{\prime}\right], t}\right) d t, \quad 0<r<r_{0} .
$$

Lemma 3.2. Let $Y$, be a pure $m$-dimensional complex space and $\phi: Y \rightarrow[-\infty, \infty)$. Then $\phi \in$ $S H(Y)$ if and only if $\phi$ is locally subharmonic in $Y$.

Proof. Using the formula (3.3), the following preliminary assertion can be proved (in the same way as in [24, Lemma 3.1]): For every pseudoball $\Delta=\Delta_{[a]}\left(R_{0}\right) \subset Y$ and for each $\phi \in L^{1}(\bar{\Delta})$, the inequality " $[\phi\rfloor \Delta]_{a, R} \geq$ (constant) $C$ for all $R \in\left(0, R_{0}\right)$ " implies that " $\left.\langle\phi\rfloor \Delta\right\rangle_{a, R} \geq C$ for all $R \in\left(0, R_{0}\right)$ ". Suppose that $\phi$ is locally subharmonic in $Y$. Let $K \subset Y$ be a compact subset and $h: K \rightarrow \mathbb{R}$ a continuous function which is semiharmonic in $\operatorname{int}(K)$ and $h(z) \geq \phi(z)$ for all $z \in \partial K$. It suffices to show that the difference $\psi:=\phi-h$ satisfies the maximum principle on each connected component $G$ of int $(K)$. Suppose now that $\sup _{G} \psi$ is attained at a point of $G$. By assumption, at each point $a \in G$ there is an open neighborhood $Q$ such that $\phi \in S H(Q)$. Then by Lemma 3.1 and the preliminary assertion, $\phi$ is solidly submedian in a neighborhood $U$ of $a \in Q$. Thus by virtue of the Gauss mean-value formula for semiharmonic functions one has $\left.\nu_{p}(a) \psi(a) \leq\langle\psi\rfloor U\right\rangle_{a, R}$ for all $R \in\left(0, R_{0}\right)$. Therefore, by the maximum principle for solidly submedian functions (Proposition 3.1), $\psi=$ (constant) $\mathrm{C}$ in $G$, so that for every sequence $\left\{a_{n}\right\} \subset G$ converging to a point $a \in \partial G$, one must have

$$
0 \geq(\phi-h)(a) \geq \limsup _{n \rightarrow \infty}(\phi-h)\left(a_{n}\right)=\mathrm{C} .
$$

Consequently $h(z) \geq \phi(z)$ for all $z \in K$, as desired.

Theorem 3.1. (1) $S M_{\mathrm{sm} . \mathrm{sph}}(Y)=S M_{\mathrm{sm} . \mathrm{sol}}(Y)=S H(Y)$. (2) If $\phi \in S H(Y)$, then $\phi$ is solidly submedian (relative to any pseudoball) at every point of $Y$.

Proof. The preliminary assertion in Lemma 3.2 shows that $S M_{\mathrm{sm} . \mathrm{sph}}(Y)$ is a subset of $S H_{\mathrm{sm} . \mathrm{sol}}(Y)$. As in the proof of Lemma 3.2, the inclusion " $S M_{\mathrm{sm} . \mathrm{sol}}(Y) \subseteq S H(Y)$ " is a consequence of Proposition 3.1. Finally the assertion (2) follows from Lemma 3.1 and the preliminary assertion in Lemma 3.2.

Remark 3.1. The second assertion of the above theorem gives a generalization of Lemma 15.2 of [7] to complex spaces. Also, it will be seen later (Theorem 3.4) that if $\phi$ is only weakly subharmonic, then the solid submean-value inequality holds almost everywhere in $Y$.

Corollary 3.1. If $\phi: Y \rightarrow \mathbb{C}$ is semiharmonic, then there exists $\psi \in C^{0}(Y) \cap S H(Y)$ such that $\psi=\phi$ almost everywhere in $Y$. 
Proof. Since $\phi$ is semiharmonic in any given pseudoball $U \subset Y$, there exists, by [24, Theorems 4.2], a continuous function $\psi_{U}$ in $U$ such that $\psi_{U}=\phi$ almost everywhere and $\psi_{U}$ has the solid mean-value property. Hence Theorem 3.1 implies that $\psi_{U}$ is subharmonic in $U$. The conclusion follows then from Lemma 3.2 .

An upper semicontinuous function $\phi: Y \rightarrow[-\infty, \infty)$ is said to be spherically (respectively, solidly) monotone at $z \in Y$ if there exists a pseudoball $U$ at $z$ of radius $r_{0}$ such that

$$
\left.[\phi\rfloor U]_{z, r} \leq[\phi\rfloor U\right]_{z, s}, \quad \text { if } 0<r \leq s<r_{0}
$$

(respectively,

$$
\left.\left.\langle\phi\rfloor U\rangle_{z, r} \leq\langle\phi\rfloor U\right\rangle_{z, s}, \quad \text { if } 0<r \leq s<r_{0}\right) .
$$

Let $p_{U}: U \rightarrow \Omega$ be an unramified Riemann covering at a point $a \in Y_{\text {reg. }}$. The radial derivative of $\phi \in C^{1}(U)$ at $z \in d U_{a}(r) \backslash p^{-1}\left(a^{\prime}\right)$ (for sufficiently small $r>0$ ) is defined by

$$
\mathrm{j}_{a, r}^{*}\left(d^{c} \phi \wedge v_{p_{U}}^{m-1}\right)(z)=\frac{r^{2 m-1}}{2}\left(\Re_{p_{U}, a} \phi\right)(z) \sigma_{a},
$$

where $\mathrm{j}_{a, r}: d U_{a}(r) \rightarrow X$, denotes the inclusion (for more detail see [24, p. 567]). The spherical mean radial derivative

$$
\left[\mathfrak{R}_{p_{U}, z}(\phi)\right]_{z, r}:=\int_{d U_{[z]}(r)} \mathfrak{R}_{p_{U}, z}(\phi) \sigma_{z}, \quad z \in U,
$$

exists for sufficiently small $r>0$. It follows from the identity (3.6) (and [24, p. 571]) that

$$
\left.\left.\frac{d}{d \rho}[\phi\rfloor U\right]_{z, \rho}\right]_{\rho=r}=\left[\Re_{p_{U}, z}(\phi)\right]_{z, r}, \quad \forall z \in U,
$$

for sufficiently small $r>0$. For a real-valued $\phi \in C^{2}\left(Y_{\text {reg }}\right)$ the spherical monotonicity at a point $a \in Y_{\text {reg }}$ amounts to the condition that $\phi$ possesses (locally) nonnegative spherical mean radial derivative at $a$, that is, there is an unramified Riemann covering $p_{U}$ at $a$ such that $\left[\Re_{p_{U}, a}(\phi)\right]_{a, r} \geq 0$ for sufficiently small $r>0$. Let $\triangle_{p_{U}}$ denote the pull-back (under $p_{U}$ ) of the Euclidean Laplace operator on $\mathbb{C}^{m}$. By differentiating under the integral sign and using the divergence theorem, it is easy to show that

$$
\left.\left.\frac{d}{d \rho}[\phi\rfloor B\right]_{a, \rho}\right]_{\rho=r}=\frac{1}{|\mathbb{S}| r^{2 m-1}} \int_{B_{[a]}(r)} \triangle_{p_{U}} \phi d v_{[a], r} .
$$

Consequently one has, for each $z \in U$,

$$
\left[\Re_{p_{U}, z}(\phi)\right]_{z, r}=\frac{r}{2 m}\left\langle\triangle_{p_{U}} \phi\right\rangle_{z, r},
$$

for sufficiently small $r>0$. 
Lemma 3.3. If $p_{U}: U \rightarrow \Omega$ is an unramified Riemann covering and $\phi \in C^{2}(U)$ is real-valued and solidly submedian, then $\triangle_{p_{U}} \phi \geq 0$.

Proof. Suppose that $\triangle_{p_{U}} \phi<0$ in $U_{[a]}\left(r_{0}\right)$ for some $a \in U$ and $r_{0}>0$. By the relations (3.7)-(3.8) one has

$$
\left.\frac{d}{d \rho}[\phi\rfloor U\right]\left._{a, \rho}\right|_{\rho=r}<0, \quad \forall r \in\left(0, r_{0}\right)
$$

Hence it follows from the relation (1.2) that

$$
\nu_{p_{U}}(a) \phi(a)>\frac{1}{|\mathbb{S}| \rho^{2 m-1}} \int_{d X_{[a]}(\rho)} \phi d \sigma_{[a], \rho}, \quad \forall \rho \in\left(0, r_{0}\right) .
$$

Thus, multiplying (3.9) by $|\mathbb{S}| \rho^{2 m-1}$ and integratingover $(0, r), 0<r<r_{0}$, yields

$$
|\mathbb{B}(r)| \nu_{p_{U}}(a) \phi(a)>\int_{X_{[a]}(r)} \phi d \tilde{v}
$$

which implies that $\nu_{p_{U}}(a) \phi(a)>\left\langle\phi\lfloor U\rangle_{a, r}\right.$, a contradiction. Therefore the Lemma is proved.

An upper semicontinuous map $\phi: Y \rightarrow[-\infty, \infty)$ is called nearly subharmonic at $z \in Y$ if there exists a pseudoball $U$ at $z$ of radius $r_{0}$ such that

$$
\left.\langle\phi\rfloor U\rangle_{z, r} \leq[\phi\rfloor U\right]_{z, r} \quad \forall r \in\left(0, r_{0}\right) ;
$$

$\phi$ is said to be seminearly subharmonic in $Y\left(\phi \in S M_{\text {sm.near }}(Y)\right.$ if there exists at each $a \in Y$ an open neighborhood $Q$ and a thin analytic subset $A_{Q}$ of $Q$ such that $\phi$ is nearly subharmonic at every point of $Q \backslash A_{Q}$. The class $\mathfrak{M}_{\text {sm.sph }}(Y)$ (respectively, $\mathfrak{M}_{\text {sm.sol }}(Y)$ ) of functions semispherically monotone (respectively, semisolidly monotone) in $Y$ is similarly defined. Note that by Lemma 3.1 and Theorem 3.1-(2), if $\phi \in S H(Y)$, then $\phi$ is spherically monotone and nearly subharmonic relative to every pseudoball at any point of $Y$.

Lemma 3.4. If $\phi$ is spherically monotone at $a \in Y$ (with $U$ and $r_{0}$ as in (3.4) for $z=a$ ), and $\phi$ is not identically equal to $-\infty$ in $U$, then the function " $r \mapsto[\phi\rfloor U]_{a, r}$ " is continuous on $\left(0, r_{0}\right)$.

Proof. Since

$$
\int_{d U_{[a]}(r)} \phi d \sigma_{[a], r}=\sum_{k=1}^{s_{a}} \int_{d V_{[a]}^{k}(r)} \phi d \sigma_{[a], r}=\sum_{k=1}^{s_{a}} \int_{\mathbb{S}_{\left[a^{\prime}\right]}(r)} \hat{\phi}_{k} d \sigma_{\left[a^{\prime}\right], r}, \quad \forall r \in\left(0, r_{0}\right),
$$

it suffices to prove that the function " $\left.r \mapsto[\phi\rfloor V^{k}\right]_{a, r}$ " is continuous on $\left(0, r_{0}\right)$. Let $\psi$ denote the induced function of $\phi\rfloor V^{k}$ on $U^{\prime}$. There exists a monotonically decreasing sequence $\left\{\psi^{(\mu)}\right\}$ of $C^{\infty}$ subharmonic functions (with increasing domains) converging pointwise on $U^{\prime}$ to $\psi$. Let $\varepsilon>0$ and $t \in\left(0, r_{0}\right)$ be given. Suppose that there exists a decreasing sequence $\left\{t_{n}\right\}$ converging to $t$ such that $\left.\left.[\psi\rfloor U^{\prime}\right]_{a^{\prime}, t}+\varepsilon \leq[\psi\rfloor U^{\prime}\right]_{a^{\prime}, t_{n}}$ for each $n$. Then, for a sufficiently large $N$,

$$
\left.\left.\left.[\psi\rfloor U^{\prime}\right]_{a^{\prime}, t}+\varepsilon \leq\left[\psi^{(\mu)}\right\rfloor U^{\prime}\right]_{a^{\prime}, t_{n}} \underset{n \rightarrow \infty}{\rightarrow}\left[\psi^{(\mu)}\right\rfloor U^{\prime}\right]_{a^{\prime}, t},
$$


where the last limit relation follows from the Stokes Theorem. Applying the monotone convergence theorem to the sequence $\left\{\psi^{(N)}-\psi^{(\mu)}\right\}$ for a sufficiently large $N$, one obtains

$$
\left.\left.\left.[\psi\rfloor U^{\prime}\right]_{a^{\prime}, t}+\varepsilon \leq \lim _{\mu \rightarrow \infty}\left[\psi^{(\mu)}\right\rfloor U^{\prime}\right]_{a^{\prime}, t}=[\psi\rfloor U^{\prime}\right]_{a^{\prime}, t},
$$

a contradiction. The conclusion now follows from the spherical monotonicity of $\phi$.

If $U$ is a pseudoball at $a_{0} \in Y$ of radius $r_{0}$, and $\phi \in L^{1}(\bar{U})$, applying the formula (3.3) to the restriction of $\phi$ to each branch of $U$, one obtains, for every $r \in\left(0, r_{0}\right)$, the relation

$$
\left.|\mathbb{B}(r)|\langle\phi\rfloor U\rangle_{a_{0}, r}=\int_{0}^{r}[\phi\rfloor U\right]_{a_{0}, t}|\mathbb{S}| t^{2 m-1} d t .
$$

Theorem 3.2. $S H(Y) \subseteq \mathfrak{M}_{\mathrm{sph}}(Y) \subseteq \mathfrak{M}_{\text {sol }}(Y)=S M_{\text {near }}(Y)$.

Proof. It will be first shown that, for a given $\phi \in C^{\text {usc }}(Y)$, "subharmonicity" implies "spherical monotonicity". Let $U$ be a pseudoball at $a \in Y$ of radius $r_{0}$ such that $\phi$ is subharmonic in $U$. Let $W_{\varepsilon}$ and the smooth approximations $\tilde{\phi}_{k, \varepsilon}$ of $\tilde{\phi}_{k}$ (extended to be 0 outside $W_{\varepsilon}$ ), $k=1, \cdots, l$, be defined as in the proof of Theorem 4.2 of [24]. It will be shown that each $\tilde{\phi}_{k, \varepsilon}$ is solidly submedian in $W_{\varepsilon}$. Let $\mathfrak{m}_{\mathbb{B}(r)}:=\frac{1}{|\mathbb{B}(r)|} \chi_{\mathbb{B}(r)}$ be the measure defined by a uniform unit mass distribution over the ball $\mathbb{B}(r)$. Take $z^{\prime} \in U^{\prime}$. There exists $r_{z^{\prime}}>0$ such that

$$
\begin{aligned}
\left.\left\langle\tilde{\phi}_{k}\right\rfloor U^{\prime}\right\rangle_{z^{\prime}, r} & =\frac{1}{|\mathbb{B}(r)|} \int_{\mathbb{B}_{\left[z^{\prime}\right]}(r)} \tilde{\phi}_{k}(y) d v(y) \\
& =\left(\mathfrak{m}_{\mathbb{B}}(r) * \tilde{\phi}_{k}\right)\left(z^{\prime}\right), \quad 0<r<r_{z^{\prime}} .
\end{aligned}
$$

Thus for every $z \in V^{j} \backslash\{a\}$,

$$
\tilde{\phi}_{k, \varepsilon}\left(z^{\prime}\right)=\left(h_{\varepsilon} * \tilde{\phi}_{k}\right)\left(z^{\prime}\right) \leq h_{\varepsilon} *\left(\mathfrak{m}_{\mathbb{B}}(r) * \tilde{\phi}_{k}\right)\left(z^{\prime}\right), \quad \forall r \in\left(0, r_{z^{\prime}}\right) .
$$

Hence (following an argument of [14, p. 205]) the inequality

$$
\tilde{\phi}_{k, \varepsilon}\left(z^{\prime}\right) \leq\left(\mathfrak{m}_{\mathbb{B}}(r) * \tilde{\phi}_{k, \varepsilon}\right)\left(z^{\prime}\right)=\left\langle\tilde{\phi}_{k, \varepsilon} \mid U^{\prime}\right\rangle_{z^{\prime}, r}
$$

holds at each $z^{\prime} \in W_{\varepsilon} \backslash\left\{a^{\prime}\right\}$ for sufficiently small $r>0$. Thus by Lemma 3.3, $\triangle_{p_{U}} \tilde{\phi}_{k, \varepsilon}$ is nonnegative in $W_{\varepsilon} \backslash\left\{a^{\prime}\right\}$, and therefore by the formulas (3.7)-(3.8), the function " $r \mapsto\left[\tilde{\phi}_{k, \varepsilon}\right]_{z^{\prime}, r}$ " is increasing on $\left(0, r_{0}-\varepsilon\right)$. Moreover, for a fixed $r \in\left(0, r_{0}\right)$,

$$
\left.\left.\left[\tilde{\phi}_{k}\right\rfloor U^{\prime}\right]_{z^{\prime}, r}=\lim _{\varepsilon \rightarrow 0}\left[\tilde{\phi}_{k, \varepsilon}\right\rfloor W_{\varepsilon}\right]_{z^{\prime}, r}, \quad \forall z^{\prime} \in U^{\prime} .
$$

It follows from this that the function " $r \mapsto[\phi\rfloor U]_{z, r}$ " is increasing on $\left(0, r_{0}\right)$ for every $z \in U$. Thus $\phi$ is spherically monotone at every point of $Y$. By approximating the last integral in the formula (3.11) by its Riemann sums, it follows easily that the "spherical monotonicity" of $\phi$ at a point $z \in Y$ implies its "solid monotonicity" at the same point.

To prove the assertion that $\phi$ is solidly monotone at a point $z \in Y$ if and only if it is nearly subharmonic at the same point, it may be assumed without loss of generality that $\phi$ is not identically 
equal to $-\infty$ in $U$. Then by Lemma 3.4, differentiation of the formula (3.11) yields for each $z \in U$ and $r \in\left(0, r_{0}\right)$,

$$
\left.\left.\left.\left.\frac{r}{2 m} \frac{d}{d \rho}\langle\phi\rfloor U\right\rangle_{z, \rho}\right]_{\rho=r}=[\phi\rfloor U\right]_{z, r}-\frac{1}{|\mathbb{B}(r)|} \int_{0}^{r}[\phi\rfloor U\right]_{z, t} t^{2 m-1} d t .
$$

Therefore the desired equivalence follows.

To prove that "spherical monotonicity" at a point implies "near subharmonicity" at the same point, suppose that (with $Q$ and $U$ as above) the inequality (3.4) holds for each $z \in Q$. Then the relation (3.11) implies that

$$
\left.|\mathbb{B}(r)|\langle\phi\rfloor U\rangle_{z, r} \leq[\phi\rfloor U\right]_{z, r} \frac{|\mathbb{S}| r^{2 m}}{2 m}, \quad \forall r \in\left(0, r_{0}\right),
$$

so that the inequality (3.13) holds, thus proving the desired claim.

An upper semicontinuous function $\psi: Y \rightarrow[-\infty, \infty)$ is called: (1) strongly regularized in $Y$, if $\psi$ satisfies both the conditions (1.1) and (1.2) for all pseudoballs $\Delta$ at every point of $Y$; (2) a Jensen function $(\psi \in J(Y)$ ), if at each $z \in Y$ the inequality (3.2) holds for $\psi$ with respect to every standard domain $\Delta=\Delta_{z}\left(R_{0}\right)$, where, in the case of a pseudoball (respectively, pseudopolydisc), $R_{0}=d_{\mathrm{pd}}(a)$ (respectively, $R_{0}=d(a)$ ). Observe that the expression [24, (3.9)] (where the proof, with slight modifications, remains valid in the case of a pseudopolydisc) shows that every continuous function $\psi: Y \rightarrow \mathbb{R}$ is strictly regularized in $Y$. This property shall be extended to Jensen (hence plurisubharmonic) functions. If $\psi: Y \rightarrow[-\infty, \infty)$, define

$$
\psi^{(*)}(a):=\limsup _{z \rightarrow a} \psi(z), \quad \forall a \in Y .
$$

Since every subharmonic function is weakly Jensen, it follows from the maximum principle (Proposition 3.1) that $\psi=\psi^{(*)}$ for all $\psi \in S H(Y)$ (compare [9, Theorem J2(c)]).

Proposition 3.2. (1) If $\psi \in S H(Y)$, then $\psi$ is strongly regularized in $Y$. In particular, if $\psi \in$ $S H(Y)$ is not identically zero, then the set $\{z \in Y \mid \psi(z) \neq 0\}$ is of positive measure at some point of $Y$. (2) If $\psi \in J(Y)$, then $\psi$ is strictly regularized in $Y$.

Proof. Consider first the case $\psi \in S H(Y)$. For any pseudoball $\Delta$ at a point $a \in Y$ and a fixed small positive $r$,

$$
\begin{aligned}
\left.\limsup _{\varepsilon \rightarrow 0}\langle\psi\rfloor \Delta\right\rangle_{a, \varepsilon} & \leq \limsup _{\varepsilon \rightarrow 0}\left\langle\sup _{z \in \Delta_{a}(r) \backslash\{a\}} \psi(z)\right\rangle_{a, \varepsilon} \\
& \left.=\lim _{\varepsilon \rightarrow 0}\langle 1\rfloor \Delta\right\rangle_{a, \varepsilon} \sup _{z \in \Delta_{a}(r) \backslash\{a\}} \psi(z) \\
& =\nu_{p_{\Delta}}(a) \sup _{z \in \Delta_{a}(r) \backslash\{a\}} \psi(z) .
\end{aligned}
$$

Here (on the above right-hand side) the relation (3.9) of [24] is invoked. Thus

$$
\left.\limsup _{\varepsilon \rightarrow 0}\langle\psi\rfloor \Delta\right\rangle_{a, \varepsilon} \leq \nu_{p_{\Delta}}(a) \psi(a) .
$$


It follows then from the solid submean-value property of $\psi$ that the limit relation (1.1) holds. The relation (1.2) can be similarly proved.

If $\psi \in J(Y)$, then the inequality (3.12) remains valid for any standard domain $\Delta$ at each point $a \in Y$, hence the limit relation (1.1) follows from the solid submean-value property (3.2)

A maximum principle for continuous nearly subharmonic functions in $\mathbb{C}^{m}$ was proved in $[6$, Lemma 3.1, p. 5]. This result is generalized and strengthened below for later applications:

Proposition 3.3. (Maximum principle for seminearly subharmonic functions) Assume $\phi$ is a realvalued, continuous, seminearly subharmonic function in a domain $D \subseteq Y$. If $\phi$ is bounded above and admits a peak point in $D$, then $\phi=$ constant in $D$.

Proof. Let $\mathcal{P}:=\{z \in D \mid \phi(z)=M\}$ be the peak set of $\phi$. There exists at every $a \in D$ an open neighborhood $Q$ and a thin analytic subset $A_{Q}$ of $Q$ such that $\phi$ is nearly subharmonic at every point of $Q \backslash A_{Q}$. Let $\hat{D}:=\cup\left(Q \backslash A_{Q}\right)$. Consider first the case $C_{0} \cap \mathcal{P} \neq \emptyset$ for some component $C_{0}$ of $\hat{D}$. Let $C_{0}$ be any such component and $U$ a pseudoball of radius $r_{0}$ at a point $a_{0} \in C_{0} \cap \mathcal{P}$ such that the inequality

$$
\left.\langle\phi\rfloor U\rangle_{z, r} \leq[\phi\rfloor U\right]_{z, r} \quad \forall r \in\left(0, r_{0}\right),
$$

holds for each $z \in U$. Observe that the mapping $r \mapsto[\phi\rfloor U]_{a_{0}, r}$ is continuous in $r$. Suppose there exists $t_{1} \in\left(0, r_{0}\right)$ such that $\left.[\phi\rfloor U\right]_{a_{0}, t_{1}}<M-\frac{1}{N}$ (for $N>\frac{1}{M}$ ). Following an idea of $[6$, p. 5 (last paragraph)], it will be shown that this assumption leads to a contradiction to the inequality (3.13). Let $\rho^{*}$ be the infimum of all $t \in\left(0, t_{1}\right)$ such that the spherical means $\left.[\phi\rfloor U\right]_{a_{0}, t}$ are bounded above by $[\phi\rfloor U]_{a_{0}, t_{1}}$. Then $\rho^{*}>0$, for, otherwise, there is a sequence $\left\{t_{\mu}\right\}$ converging to 0 with $\left.[\phi\rfloor U]_{a_{0}, t_{\mu}} \leq[\phi\rfloor U\right]_{a_{0}, t_{1}}$ for each $\mu$, such that, by virtue of [24, Proposition 3.1],

$$
\left.\operatorname{deg}\left(p_{U}\right) \phi\left(a_{0}\right) \leq[\phi\rfloor U\right]_{a_{0}, t_{1}}<M-\frac{1}{N} .
$$

Hence a contradiction results. The Fubini type formula (3.3) implies that

$$
\begin{aligned}
\left.\left|\mathbb{B}\left(\rho^{*}\right)\right|\langle\phi\rfloor U\right\rangle_{a_{0}, \rho^{*}} & =\sum_{j=1}^{l} \int_{V_{\left[a_{0}\right]}^{j}\left(\rho^{*}\right)} \phi d v_{\left[a_{0}\right]} \\
& =\int_{0}^{\rho^{*}}\left(\sum_{j=1}^{l} \int_{\mathbb{S}_{\left[a_{0}^{\prime}\right]}(t)} \tilde{\phi}_{j} d \sigma_{\left[a_{0}^{\prime}\right], t}\right) d t \\
& \left.\left.=\int_{0}^{\rho^{*}}[\phi\rfloor U\right]_{a_{0}, t}|\mathbb{S}| t^{2 m-1} d t>[\phi\rfloor U\right]_{a_{0}, t_{1}} \frac{|\mathbb{S}|\left(\rho^{*}\right)^{2 m}}{2 m} .
\end{aligned}
$$

This leads to a contradiction $\left.\langle\phi\rfloor U\rangle_{a_{0}, \rho^{*}}>[\phi\rfloor U\right]_{a_{0}, \rho^{*}}$. Therefore one must have $\left.[\phi\rfloor U\right]_{a_{0}, r} \geq M$ for every $r \in\left(0, r_{0}\right)$. Then the formula (3.11) yields that $\left.\langle\phi\rfloor U\right\rangle_{a_{0}, r} \geq M$ for such $r$. Since $\phi \leq M$, this implies that $\phi=M$ in a neighborhood of $a_{0}$. Thus $C_{0} \subseteq \mathcal{P}$. Consequently, by the continuity of $\phi, \phi=M$ also in $D$. 
Now suppose that $C_{0} \cap \mathcal{P}=\emptyset$ for all components $C_{0}$ of $\hat{D}$. Let $z_{*}$ be any point of $\mathcal{P}$. Then $z_{*} \in A_{Q}$ for every $Q$ (chosen as above). There exists a sequence $\left\{a_{n}\right\}$ in $Q \backslash\left(A_{Q} \cup \mathcal{P}\right)$ (for a fixed $Q$ ) converging to $z_{*}$. Choose a sequence of pseudoballs $U_{\left[a_{n}\right]}\left(r_{n}\right)$ such that $\phi$ is nearly submedian at each point of $U_{n}=U_{\left[a_{n}\right]}\left(r_{n}\right)$. Then (as shown in the equation (3.14)),

$$
\left.\operatorname{deg}\left(p_{U}\right) \phi\left(a_{n}\right) \leq[\phi\rfloor U_{n}\right]_{a_{n}, t_{n}}<M-\frac{1}{N},
$$

(where $t_{n}$ depends on $a_{n}$ ). Thus by the continuity of $\phi$, one has $\phi\left(z_{*}\right)<M$, a contradiction! Hence the preceding argument showing that $\phi=M$ in a neighborhood of $a_{0}$ implies the same for $a_{n}$. Consequently the sequence $\left\{a_{n}\right\}$ lies in $\mathcal{P}$, again a contradiction! Therefore $C_{0} \cap \mathcal{P} \neq \emptyset$ for some component $C_{0}$ of $\hat{D}$, and thereby the proposition is proved.

Theorem 3.3. For a continuous function $\phi: Y \rightarrow \mathbb{R}$ the following conditions are equivalent: (i) $\phi \in \mathfrak{M}_{\mathrm{sm} . \operatorname{sph}}(Y)$; (ii) $\phi \in \mathfrak{M}_{\text {sm.sol }}(Y)$; (iii) $\phi \in S M_{\text {sm.near }}(Y)$; (iv) $\phi \in S H(Y)$.

Proof. In view of Theorem 3.2, it suffices to prove that every element $\phi \in S M_{\text {sm.near }}(Y)$ is subharmonic in $Y$. Let $K \subset Y$ be a compact set and $h: K \rightarrow \mathbb{R}$ a continuous function such that $h$ is semiharmonic in $\operatorname{int}(K)$ and $h(z) \geq \phi(z)$ for every $z \in \partial K$. Suppose that $\phi$ is seminearly subharmonic in $Y$. Then at each point $a$ in a connected component $G$ of $\operatorname{int}(K)$, there is an open neighborhood $Q$ and a thin analytic subset $A_{Q}$ of $Q$ such that $\phi$ is nearly subharmonic at each point of $Q \backslash A_{Q}$. Thus given $z \in Q \backslash A_{Q}$, there is a pseudoball $U=U_{z}\left(r_{0}\right)$ in which the inequality (3.13) holds, and, consequently,

$$
\left.\langle\phi-h\rfloor U\rangle_{z, r} \leq[\phi-h\rfloor U\right]_{z, r}, \quad \forall r \in\left(0, r_{0}\right) .
$$

Thus by Proposition 3.3, the function $\phi-h$ satisfies the maximum principle on $G$. It follows that $\phi-h \leq 0$ in $\operatorname{int}(K)$. This proves the subharmonicity of $\phi$.

A locally Lipschitz function is semiharmonic if it has almost everywhere vanishing local radial derivative (this will be shown in a later work). With $C^{2}$-differentiability, "subharmonicity" betokens similarly the nonnegativity (off a thin analytic subset) of the local spherical mean radial derivative:

Proposition 3.4. (1) Let $\phi \in C^{0}(Y) \cap C^{2}\left(Y_{\text {reg }}\right)$ be real-valued. Assume that for some thin analytic subset $A$ of $Y$ there exists, at each $a \in Y \backslash A$, an unramified Riemann covering $p_{U}: U \rightarrow \Omega$ satisfying one of the following: (a) $\phi$ has (locally) nonnegative spherical mean radial derivative at each point of $U$; (b) $\triangle_{p_{U}} \phi \geq 0$ in $U$. Then $\phi$ is subharmonic in $Y$. (2) If $\phi \in \mathcal{S} H(Y) \cap C^{2}\left(Y_{\mathrm{reg}}\right)$, then both the conditions (a) and (b) are valid for any unramified Riemann covering (on an open set in $Y_{\text {reg}}$ ).

Proof. Let $p_{U}: U \rightarrow \Omega$ be an unramified Riemann covering at $a \in Y_{\text {reg. Clearly for any } \phi \in C^{2}(U)}$ the relation (3.8) implies that the conditions (a) and (b) are equivalent; moreover, by virtue of the formula (3.7) each of these implies that $\phi$ is spherically monotone in $U$. Thus the first assertion follows from Theorem 3.3. Since each subharmonic function is solidly submedian, the second assertion is a consequence of the preceding and Lemma 3.3.

Definition 3.1. A locally integrable function $\phi: Y \rightarrow[-\infty, \infty)$ is called weakly subharmonic in $Y\left(\phi \in \mathcal{S H}_{\mathfrak{w}}(Y)\right)$ if there exists at each $a \in Y$ a standard domain $\Delta=\Delta_{a}(R)$ such that for all nonnegative $u \in C_{0}^{2}(\Delta)$, 


$$
\int_{\Delta_{a}(r)} \phi d d^{c} u \wedge v_{p_{U}}^{m-1} \geq 0, \quad \forall r \in(0, R) .
$$

Observe that, if $p: D \rightarrow \Omega$, is a Riemann semicovering on an open set $D \subseteq Y$ and if $\phi \in$ $\mathcal{S H}_{\mathfrak{w}}(D)$, then the inequality (3.15) holds with $D$ in place of $\Delta_{a}(r)$ for all nonnegative $u \in C_{0}^{2}(D)$.

Theorem 3.4. (1) There exists a bijection between $\mathcal{S H}_{\mathfrak{w}}(Y)$ and $S H_{\{\not \equiv-\infty\}}(Y)$ taking each $\phi \in$ $\mathcal{S H}_{\mathfrak{w}}(Y)$ to a unique element $\hat{\phi} \in S H_{\{\neq-\infty\}}(Y)$ such that $\hat{\phi}=\phi$ almost everywhere in $Y$. (2) A function $\phi: Y \rightarrow[-\infty, \infty)$ is weakly subharmonic and regularized in $Y$ if and only if $\phi \in$ $S H_{\{\not \equiv-\infty\}}(Y)$.

Proof. Suppose that $\phi \in \mathcal{S H}_{\mathfrak{w}}(Y)$. Let $a \in Y$ and $\tilde{\phi}_{k, \varepsilon}$ be defined as in the proof of Theorem 3.2 (for $U=\Delta$ as in the above definition). The weak subharmonicity of $\phi$ implies that the induced function $\tilde{\phi}_{k} \in \mathcal{S H}_{\mathfrak{w}}\left(\Delta^{\prime}\right)$, hence the function $\tilde{\phi}_{k, \varepsilon}$ is weakly subharmonic in $W_{\varepsilon}$ for every $\varepsilon>0$ (by the proof of the assertion "(1) implies (2)" in [24, Theorem 4.2, p. 564]). Suppose that the form $d d^{c} \tilde{\phi}_{k, \varepsilon} \wedge v_{\left[a^{\prime}\right]}^{m-1}$ is negative at some $z^{\prime} \in W_{\varepsilon}$. Then $d d^{c} \tilde{\phi}_{k, \varepsilon} \wedge v_{\left[a^{\prime}\right]}^{m-1}<0$ in a neighborhood $Q \subseteq W_{\varepsilon}$ of $z^{\prime}$, hence for all $u \in C_{0}^{2}(Q)$ with $u \geq 0$ and $u=1$ in a neighborhood of $z^{\prime}$, one has

$$
\int_{\Delta^{\prime}} \tilde{\phi}_{k, \varepsilon} d d^{c} u \wedge v_{\left[a^{\prime}\right]}^{m-1}=\int_{\Delta^{\prime}} u d d^{c} \tilde{\phi}_{k, \varepsilon} \wedge v_{\left[a^{\prime}\right]}^{m-1}<0,
$$

a contradiction. Thus $\triangle_{\mathrm{id}} \tilde{\phi}_{k, \varepsilon} \geq 0$ in $W_{\varepsilon}$, so that (by the proof of Theorems 3.2) the function $\tilde{\phi}_{k, \varepsilon}$ is spherically monotone, and for every $z^{\prime} \in W_{\varepsilon} \backslash\left\{a^{\prime}\right\}$,

$$
\tilde{\phi}_{k, \varepsilon}\left(z^{\prime}\right) \leq\left\langle\tilde{\phi}_{k, \varepsilon}\right\rangle_{z^{\prime}, r}
$$

for sufficiently small $r>0$. It follows from the argument of [13, p. 20] that the function $\varepsilon \mapsto \tilde{\phi}_{k, \varepsilon}\left(z^{\prime}\right)$ is nondecreasing for fixed $z^{\prime} \in W_{\varepsilon}$. Define

$$
\psi_{\Delta}(z):=\min _{k} \lim _{\varepsilon \rightarrow 0}\left\{\tilde{\phi}_{k, \varepsilon}\left(z^{\prime}\right) \mid V^{k} \ni z\right\}, \quad \forall z \in \Delta .
$$

Then $\psi_{\Delta}$ is upper semicontinuous in $\Delta$. Moreover $\psi_{\Delta}(z)=\phi(z)$ for almost every $z \in \Delta$. Also, the formula (3.17) implies that for each $z \in \Delta$,

$$
\begin{aligned}
\psi_{\Delta}(z) & \leq \min _{k}\left\{\tilde{\phi}_{k, \varepsilon}\left(z^{\prime}\right) \mid V^{k} \ni z\right\} \\
& \leq \min _{k} \frac{1}{|\mathbb{B}(r)|} \int_{\mathbb{B}_{\left[z^{\prime}\right]}(r)} \tilde{\phi}_{k, \varepsilon}(y) d v(y),
\end{aligned}
$$

for sufficiently small positive $\varepsilon$ and $r$, where the last inequality follows from (3.16). Letting $\varepsilon \rightarrow 0$ this inequality implies that

$$
\nu_{p}(z) \psi_{\Delta}(z) \leq\left\langle\psi_{\Delta}\right\rangle_{z, r}
$$


for sufficiently small $r>0$. Therefore by Theorem 3.1, $\psi_{\Delta} \in S H_{\{\neq-\infty\}}(\Delta)$. It follows from Proposition 3.2 that setting $\psi:=\psi_{\Delta}$ on each $\Delta$ (as above) defines a subharmonic function $\psi: Y \rightarrow[-\infty, \infty)$ which coincids with $\phi$ almost everywhere in $Y$.

Conversely, given $\hat{\phi} \in S H_{\{\neq-\infty\}}(Y)$, it will first be shown that $\hat{\phi}$ is locally integrable in $Y$. For this purpose a preliminary observation is needed: if $U_{z}(r) \subset Y, 0<r<d(z)$, is a pseudoball at $z$ and $\hat{\phi}(z)>-\infty$, then $\hat{\phi}$ is integrable over $U_{z}(r)$. This is an immediate consequence of the local solid submean-value inequality for $\hat{\phi}$. Let $I$ be the set of all points of $Y$ where $\hat{\phi}$ is locally integrable. Clearly $I$ is an open set and since locally $\hat{\phi} \not \equiv-\infty$, it follows from the preliminary observation that $I$ is nonempty. On the other hand, if $b \in Y \backslash I$, the preliminary observation implies that $\hat{\phi} \equiv-\infty$ almost everywhere in some pseudoball $U_{b}(R)$; for, otherwise, $\hat{\phi}(z)>-\infty$ for some point $z \in U_{b}(r)$ for each (sufficiently small) $r>0$; hence there is a sequence $\left\{z_{n}\right\} \subset Y$ converging to $b$ such that $\hat{\phi}\left(z_{n}\right)>-\infty$ for every $n$. If $0<R<d(b) / 2$ and $n$ is sufficiently large, one has $\left\|z_{n}-b\right\|<R$ and $\hat{\phi}$ is locally integrable over $\bar{U}_{z_{n}}(R)$; but since $b \in U_{z_{n}}(R)$, it follows that $b \in I$, a contradiction. Thus $U_{b}(R) \subset Y \backslash I$, so that $Y \backslash I$ is open. Applied to each component $Y_{\mu}$ of $Y$, this implies that $Y_{\mu} \backslash I=\emptyset$, hence $\hat{\phi}$ is locally integrable in $Y$.

It remains to show that $\hat{\phi}$ satisfies the condition (3.15). Let $\Delta$ be a standard domain at $a \in Y$ and denote by $\tilde{\phi}^{k}$ the function on $\Delta^{\prime}$ induced by $\left.\hat{\phi}\right\rfloor V^{k}$. Then $\tilde{\phi}^{k}$ is subharmonic, hence solidly submedian, in $\Delta^{\prime}$. It follows as in the proof of Theorem 3.2 that the smooth approximations $\tilde{\phi}_{\varepsilon}^{k}$ of $\tilde{\phi}^{k}$ are solidly submedian in $W_{\varepsilon}$. Consequently by Lemma $3.3, \triangle_{\mathrm{id}} \tilde{\phi}_{\varepsilon}^{k} \geq 0$ in $W_{\varepsilon}^{\prime}$ (for a given open set $\left.\Delta^{\prime \prime} \Subset \Delta^{\prime}\right)$. Then by the $L^{1}$-convergence of $\tilde{\phi}_{\varepsilon}^{k}$ to $\tilde{\phi}_{k}$, for each nonnegative $g \in C_{0}^{2}\left(\Delta^{\prime}\right)$,

$$
\int_{\Delta^{\prime}} \tilde{\phi}^{k} d d^{c} g \wedge v_{\left[a^{\prime}\right]}^{m-1}=\lim _{\varepsilon \rightarrow 0} \int_{\Delta^{\prime}} g d d^{c} \tilde{\phi}_{\varepsilon}^{k} \wedge v_{\left[a^{\prime}\right]}^{m-1} \geq 0 .
$$

Let $u \in C_{0}^{2}(\Delta)$ with $u \geq 0$. Since the function $\tilde{u}^{k}$ induced by $\left.u\right\rfloor V^{k}$ has compact support in $\Delta^{\prime}$, there exists a sequence $f_{n} \in C_{0}^{2}\left(\Delta^{\prime}\right)$ such that $f_{n} \rightarrow \triangle_{\mathrm{id}} \tilde{u}^{k}$ in $L^{1}\left(\Delta^{\prime}\right)$. By solving the Dirichlet problem

$$
d d^{c}\left(w v_{\left[a^{\prime}\right]}^{m-1}\right)=\frac{1}{4 m} f_{n} v_{\left[a^{\prime}\right]}^{m} \text { in } \Delta^{\prime}, \quad w=0 \text { on } \partial D_{n},
$$

where $D_{n}$ is an open neighborhood of $\operatorname{Spt}\left(f_{n}\right)$ with smooth boundary, one obtains a sequence $\left\{w_{n}\right\}$ in $C_{0}^{2}\left(\Delta^{\prime}\right)$ such that $\triangle_{\mathrm{id}} w_{n}$ tends to $\triangle_{\mathrm{id}} \tilde{u}^{k}$ in $L^{1}\left(\Delta^{\prime}\right)$. Without loss of generality it may be assumed that each $w_{n}$ is nonnegative. Then by the inequality (3.18),

$$
\int_{V^{k}} \hat{\phi} d d^{c} u \wedge v_{p}^{m-1}=\int_{\Delta^{\prime} \backslash\left\{a^{\prime}\right\}} \tilde{\phi}^{k} d d^{c} \tilde{u}^{k} \wedge v_{\left[a^{\prime}\right]}^{m-1} \geq 0
$$

The proof of the assertion (1) is thereby completed. The second assertion follows from the preceding proof and the regularity of a subharmonic function.

\section{Plurisubharmonicity}

Let $\tilde{\pi}: \mathbb{C}^{m} \backslash\{0\} \rightarrow \mathbb{P}^{m-1}(\mathbb{C})$ denote the natural projection. The induced map $\pi: \mathbb{S}^{2 m-1} \rightarrow \mathbb{P}^{m-1}(\mathbb{C})$ defines a fiber bundle with fiber $\pi^{-1}([\mathfrak{a}])=\mathfrak{l}_{\mathfrak{a}}:=\{\zeta \mathfrak{a} \mid \zeta \in \mathbb{C},\|\zeta\|=1\}$ over $[\mathfrak{a}] \in \mathbb{P}^{m-1}(\mathbb{C})$, where 
$\|\mathfrak{a}\|=1$. Let $\mathfrak{j}_{(r)}: \mathbb{S}^{2 m-1} \rightarrow \mathbb{C}^{m}$ be the dilation: $\mathfrak{j}_{(r)}(\mathfrak{z})=r \mathfrak{z}$. The Fubini-Study form $\ddot{\omega}$ on $\mathbb{P}^{m-1}(\mathbb{C})$ pulls back under $\tilde{\pi}$ to the projective form $\omega:=d d^{c} \log \|z\|^{2}$ on $\mathbb{C}^{m} \backslash\{0\}$. Thus

$$
\pi^{*}\left(\ddot{\omega}^{m-1}\right)=\mathfrak{j}_{(r)}^{*}\left(\tilde{\pi}^{*}\left(\ddot{\omega}^{m-1}\right)\right)=\mathfrak{j}_{(r)}^{*}\left(\omega^{m-1}\right) .
$$

An upper semicontinuous function $\phi: Y \rightarrow[-\infty, \infty)$ is said to be (locally) radially submedian $\left(\phi \in S M_{\mathrm{rad}}(Y)\right)$ if there exists at every point $a \in Y$ a pseudoball $U$ of radius $r_{0}$ such that for all $\mathfrak{a} \in \mathbb{S}^{2 m-1}$,

$$
\nu_{p_{U}}(a) \phi(a) \leq \frac{1}{2 \pi} \int_{0}^{2 \pi} \sum_{k} \tilde{\phi}_{k}\left(a^{\prime}+r e^{i \theta} \mathfrak{a}\right) d \theta, \quad 0<r<r^{0},
$$

(where the sum ranges over all branches of $U$ ) for some $r^{0}=r^{0}(a, \mathfrak{a}) \leq r_{0}$. An upper semicontinuous function $\phi: Y \rightarrow[-\infty, \infty)$ is said to be (locally) semiradially submedian $\left(\phi \in S_{\mathrm{sm} . \mathrm{rad}}(Y)\right)$ if $\phi$ belongs, locally at every $a \in Y$, to $S M_{\mathrm{rad}}\left(Q \backslash A_{Q}\right)$ for some thin analytic subset $A_{Q}$ in an open neighborhood $Q$ of $a$. A peak point of $\phi\rfloor Q$ belonging to $A_{Q}$ is called a local exceptional peak point.

Proposition 4.1. $P S H(Y) \subseteq S M_{\mathrm{rad}}(Y) \subseteq S H(Y)$.

Proof. Let $U$ be a pseudoball at $a \in Y$. The first inclusion relation follows from the fact that given $\phi \in \operatorname{PSH}(Y)$, each induced function $\tilde{\phi}_{k}$ is subharmonic at $a_{0}^{\prime}$ when restricted to the complex line $\mathfrak{L}_{\mathfrak{a}}=\left\{z \in \mathbb{C}^{m} \mid z=a_{0}^{\prime}+\zeta \mathfrak{a}, \zeta \in \mathbb{C}\right\}$, for each $\mathfrak{a} \in \mathbb{S}^{2 m-1}$. To prove the second inclusion relation, suppose that $\phi \in S M_{\mathrm{rad}}(Y)$. The expression (4.1) allows one to evaluate the spherical means of each $\tilde{\phi}_{k}: U^{\prime} \rightarrow \mathbb{R}$ by integrating along fibers of the bundle map $\pi$ (as in [20, p. 202]). The detail is given below for completeness.

Without loss of generality assume that $a_{0}^{\prime}=0$. Write $\mathfrak{z}=e^{i \theta} \mathfrak{a}, \mathfrak{a} \in \mathbb{S}^{2 m-1}, 0 \leq \theta \leq 2 \pi$. Let $\mathfrak{i}_{\mathfrak{a}}: \mathfrak{L}_{\mathfrak{a}} \hookrightarrow \mathbb{C}^{m}, \mathfrak{j}_{\mathfrak{a}}: \mathfrak{l}_{\mathfrak{a}} \hookrightarrow \mathfrak{L}_{\mathfrak{a}}, \mathfrak{J}_{\mathfrak{a}}: \mathfrak{l}_{\mathfrak{a}} \hookrightarrow \mathbb{S}^{2 m-1}$ be the inclusion mappings. Then

$$
\mathfrak{J}_{\mathfrak{a}}^{*} \mathfrak{j}_{(r)}^{*}\left(d^{c} \ln \|z\|^{2}\right)=\mathfrak{j}_{\mathfrak{a}}^{*} \mathfrak{i}_{\mathfrak{a}}^{*}\left(d^{c} \ln \|z\|^{2}\right)=\mathfrak{j}_{\mathfrak{a}}^{*}\left(d^{c} \ln \|\zeta\|^{2}\right)=\frac{1}{2 \pi} d \theta
$$

Therefore for small $r>0$ one has

$$
\begin{aligned}
\nu_{p}\left(a_{0}\right) \phi\left(a_{0}\right) & =\int_{[\mathfrak{a}] \in \mathbb{P}^{m-1}(\mathbb{C})} \nu_{p}\left(a_{0}\right) \phi\left(a_{0}\right) \ddot{\omega}^{m-1} \\
& \leq \int_{[\mathfrak{a}] \in \mathbb{P}^{m-1}(\mathbb{C})}\left[\int_{0}^{2 \pi} \sum_{j=1}^{l} \tilde{\phi}_{j}\left(a_{0}^{\prime}+r e^{i \theta} \mathfrak{a}\right) \frac{d \theta}{2 \pi}\right] \ddot{\omega}^{m-1} \\
& =\sum_{j=1}^{l} \int_{\mathbb{S}^{2 m-1}} \tilde{\phi}_{j}\left(a_{0}^{\prime}+r \mathfrak{z}\right) \mathfrak{j}_{r}^{*}\left(\sigma_{0}\right)=\sum_{j=1}^{l} \int_{d V_{\left[a_{0}\right]}^{j}(r)} \phi \sigma_{a_{0}} \\
& =[\phi] U]_{a_{0}, r}
\end{aligned}
$$

Thus $\phi$ is spherically submedan in $Y$ Therefore, by Theorem 3.1, $\phi \in S H(Y)$.

Theorem 4.1. (1) If $\phi \in S M_{\text {sm.rad }}(Y)$ and is continuous at every local exceptional peak point (if any), then $\phi$ is subharmonic. (2) If $Y$ is a normal space, then $\operatorname{SM}_{\mathrm{sm} . \operatorname{rad}}(Y)=P S H(Y)$. 
Proof. By Proposition 4.1 and Theorem 3.1, a function $\phi \in S M_{\mathrm{sm} . r a d}(Y)$ that is continuous at every local exceptional peak point is semisolidly submedian, hence subharmonic, in $Y$. To prove the second assertion, note that in the definition of the set $S M_{\mathrm{sm} . r a d}(Y)$, the local analytic set $A_{Q}$ at a point $z$ can be chosen (without loss of generality) so that $Q \backslash A_{Q} \subseteq Q_{\text {reg. }}$. The desired conclusion follows then from the plurisubharmonicity test in $\mathbb{C}^{m}([26$, p. 73$])$ and the extension theorem of Grauert and Remmert for plurisubharmonic functions on a normal complex space ([8, Satz 3, p. 181]).

A function $\phi: Y \rightarrow[0, \infty)$ is called logarithmically subharmonic (respectively, logarithmically plurisubharmonic), if $\log \phi \in S H(Y)$ (respectively, $\log \phi \in P S H(Y)$; for $C^{2}$-functions in $\mathbb{C}^{m}$, another definition of logarithmic plurisubharmonicity is given in [3, p. 200]). If $f=\left(f_{1}, \cdots, f_{N}\right): Y \rightarrow \mathbb{C}^{N}$ and $c=\left(c_{1}, \cdots, c_{N}\right)$, where each $c_{j}$ is an arbitrary positive constant, set

$$
\lfloor f\rfloor^{c}:=\left\|f_{1}\right\|^{c_{1}}+\cdots+\left\|f_{N}\right\|^{c_{N}} .
$$

If $f$ is a holomorphic map, then the function $\lfloor f\rfloor^{c}$ is logarithmically plurisubharmonic (see Example 6.3 of the Appendix); furthermore, $\log \lfloor f\rfloor^{c}$ is a Jensen function, provided $f$ has thin zero set. This is in fact a special case of the next proposition (which generalizes the classical Jensen's inequality):

Proposition 4.2. $P S H(Y)_{\{\not \equiv-\infty\}} \subseteq J(Y)$.

Proof. Let $\phi \in P S H_{\{\neq-\infty\}}(Y)$. By Theorems 4.1 and 3.4, $\phi$ is subharmonic and locally integrable in $Y$. The submean-value inequality relative to pseudoballs follows from Theorem 3.1. Suppose now that $U=\Delta_{a}(R) \Subset Y$ is a pseudopolydisc of polyradius $R$. Each induced function $\tilde{\phi}_{k}$ is plurisubharmonic in $p(U) \backslash\left\{a^{\prime}\right\}$, hence also in $p(U)$ (by [8, Satz 3, p. 181]), so that it is subharmonic in each variable $z_{j}, 1 \leq j \leq m$. Repeated integration yields the inequality

$$
\phi(a) \leq \operatorname{vol}\left(\Delta\left(a^{\prime}, R\right)\right)^{-1} \int_{\Delta\left(a^{\prime}, R\right)} \tilde{\phi}_{k}\left(z^{\prime}\right) d v\left(z^{\prime}\right) .
$$

From this and the identity (2.1) the the submean-value inequality (3.2) follows.

Definition 4.1. A locally integrable function $\psi: Y \rightarrow[-\infty, \infty)$ is said to be weakly plurisubharmonic in $Y\left(\psi \in \mathcal{P} \mathcal{S H}_{\mathfrak{w}}(Y)\right)$ if at each point $a \in Y$ there exists a standard domain $\Delta$ such that the Levi form of $\psi, \mathcal{L}(\psi, \lambda)$, is positive semidefinite in $\Delta$, that is, for some thin analytic subset $A$ of $\Delta$ (with $\left.\Delta \backslash A \subseteq \Delta^{*}\right)$ and for all nonnegative $u \in C_{0}^{2}(\Delta)$,

$$
\langle\mathcal{L}(\psi, \lambda), u\rangle_{\Delta}:=\int_{\Delta \backslash A} \psi \sum_{\mu, \nu} \frac{\partial^{2} u}{\partial p_{\mu} \partial \bar{p}_{\nu}} \lambda_{\mu} \bar{\lambda}_{\nu} d \tilde{v} \geq 0, \quad \forall \lambda \in \mathbb{C}^{m} .
$$

Similarly, a locally integrable function $\psi: Y \rightarrow[-\infty, \infty)$ is said to be weakly pluriharmonic in $Y\left(\psi \in \mathcal{P} \mathcal{H}_{\mathfrak{w}}(Y)\right)$ if at each $a \in Y$ there exists a standard domain $\Delta$ in which the above Levi form $\mathcal{L}(\psi, \lambda) \equiv 0$.

Theorem 4.2. (Compare [13, Theorem 3, p. 21] and [9, Theorem K15]) (1) $P S H_{\{\neq-\infty\}}(Y) \subseteq$

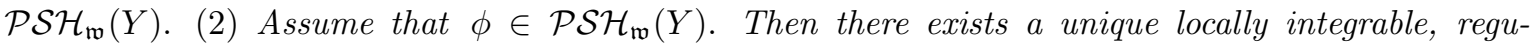
larized function $\psi: Y \rightarrow[-\infty, \infty)$ such that $\psi=\phi$ almost everywhere and $\psi \in S M_{\mathrm{rad}}(Y) \cap$ $P S H_{\{\neq-\infty\}}\left(Y_{\text {reg }}\right)$. 
Proof. Let $\phi \in P S H_{\{\neq-\infty\}}(Y)$. By Theorems 4.1 and 3.4, $\phi$ is locally integrable in $Y$. Consider first the special case of a $C^{2}$-functions $\phi: Y \rightarrow[-\infty, \infty)$. Suppose that $Y$ is nonsingular. For any standard domain $\Delta \subset Y$ and $\alpha \in C^{2}(\Delta)$, denote by $\tilde{\alpha}$ the function on $\Delta^{\prime}$ induced by $\alpha$ and set

$$
L(\alpha, \lambda)(z):=\sum_{\mu, \nu} \frac{\partial^{2} \tilde{\alpha}}{\partial z_{\mu} \partial \bar{z}_{\nu}}\left(z^{\prime}\right) \lambda_{\mu} \bar{\lambda}_{\nu}, \quad z^{\prime} \in \Delta^{\prime}, \lambda \in \mathbb{C}^{m}
$$

Then $\phi \in P S H(U)$ if and only if $L(\phi, \lambda)(z) \geq 0$ for every $z \in \Delta$ and every $\lambda \in \mathbb{C}^{m}$; but that is clearly equivalent to the requirement that there exists a thin analytic subset $A$ of $\Delta$ such that for all nonnegative $u \in C_{0}^{2}(\Delta)$,

$$
\int_{\Delta \backslash A} u L(\phi, \lambda) v_{p}^{m} \geq 0 .
$$

By [9, Lemma K14], the latter condition is in turn equivalent to the requirement (4.3) (with $D=\Delta$ ). If $Y$ is singular, then by using local desingularizations of $Y$, it is easily seen that a $C^{2}$-function $\phi$ : $Y \rightarrow \mathbb{R}$ belongs to $P S H(Y)$ precisely when it satisfies the condition (4.3) of weak plurisubharmonicity. Thus the $C^{2}$ case of the assertion (1) is established.

For the general case, observe that each induced function $\tilde{\phi}_{k}: \Delta^{\prime} \rightarrow \mathbb{R}$ is plurisubharmonic in $U^{\prime}:=\Delta^{\prime}$, hence so are the smooth approximations $\tilde{\phi}_{k, \varepsilon}$ in $W_{\varepsilon}$. Let $u \in C_{0}^{2}(\Delta)$ and denote by $\tilde{u}_{k}$ the function induced by $u\rfloor V^{k}$ in $\Delta^{\prime}$. Since the support of $\tilde{u}_{k}$ lies in $W_{\varepsilon}$ whenever $\varepsilon$ is sufficiently small, and for such values $\varepsilon$ it follows from the first part of the proof that

$$
\int_{\Delta^{\prime}} \tilde{\phi}_{k, \varepsilon} L\left(\tilde{u}_{k}, \lambda\right) d v(z) \geq 0, \quad \lambda \in \mathbb{C}^{m} .
$$

As $\varepsilon \rightarrow 0$, the function $\tilde{\phi}_{k, \varepsilon}$ converges to $\tilde{\phi}_{k}$ in $L^{1}$-norm on the support of $\tilde{u}_{k}$. Consequently $\phi$ satisfies the condition (4.3). Thus the assertion (1) is established in the general case.

Now suppose that $\phi \in \mathcal{P S H}_{\mathfrak{w}}(Y)$. Let $\Delta$ be a pseudoball at $a \in Y$ relative to which the condition (4.3) holds. Define $\psi_{\Delta}: \Delta \rightarrow[-\infty, \infty)$ by

$$
\left.\nu_{p_{\Delta}}(z) \psi_{\Delta}(z):=\lim _{r \rightarrow 0} \sum\left\{\langle\phi\rfloor V^{k}\right\rangle_{z, r} \mid V^{k} \ni z\right\}, \quad \forall z \in \Delta .
$$

Then $\psi_{\Delta}$ is regularized in $\Delta$ and $\psi_{\Delta}=\phi$ almost everywhere; moreover, the Levi form $\mathcal{L}\left(\psi_{\Delta}, \lambda\right) \geq 0$ in $\Delta$. Consider the smooth approximations $\tilde{\psi}_{k, \varepsilon}$ in $W_{\varepsilon}$ of the induced function $\tilde{\psi}_{k}$ of $\left.\psi_{k}:=\psi_{\Delta}\right\rfloor V^{k}$. The second half of the proof of [9, Theorem K15] shows that $\tilde{\psi}_{k}$ is plurisubharmonic in $\Delta^{\prime} \backslash\left\{a^{\prime}\right\}$, hence also in $\Delta^{\prime}$ (by Grauert and Remmert's extension theorem [8, Satz 3, p. 181]). It follows from Proposition 3.2 that the function $\psi$ given by $\psi:=\psi_{\Delta}$ on each $\Delta$ is well-defined. Also, $\psi$ is locally integrable, radially submedian and regularized in $Y$. Since $\psi_{\Delta}$ is plurisubharmonic in each open set $\Delta \backslash\{a\}$, so is the function $\psi$ in $Y_{\text {reg. }}$.

Remark 4.1. The above theorem and its proof (of the first assertion) imply that, if $D \subseteq Y$ is a normal, open subset and $\phi \in P S H_{\{\neq-\infty\}}(D)$, then for any Riemann semicovering $p: D \rightarrow \Omega$, the inequality (4.3) remains valid: $\langle\mathcal{L}(\phi, \lambda), u\rangle_{D} \geq 0$ for all nonnegative $u \in C_{0}^{2}(D)$ and every $\lambda \in \mathbb{C}^{m}$. 
Corollary 4.1. Let $Y$ be a normal space. A function $\phi: Y \rightarrow[-\infty, \infty)$ is weakly plurisubharmonic and regularized in $Y$ if and only if $\phi \in P S H_{\{\neq-\infty\}}(Y)$.

Proof. Suppose that $\phi \in \mathcal{P S H}_{\mathfrak{w}}(Y)$ is regularized in $Y$. Then by Theorem 4.2, $\phi$ is plurisubharmonic in $Y_{\text {reg. }}$. The Grauert and Remmert's extension theorem ([8, Satz 3, p. 181]) asserts that $\left.\phi\right\rfloor Y_{\text {reg }}$ admits a plurisubharmonic extension $\psi$ to $Y$, which, by subharmonicity, must coincide with $\phi$ (Proposition 3.2). Conversely, every element of $\mathrm{PSH}_{\{\not \equiv-\infty\}}(Y)$ is weakly plurisubharmonic and regularized in $Y$ by Theorem 4.2-(1) and Proposition 3.2.

Remark 4.2. (Compare [9, Corollary K18]) Let $Y$ be a normal space. A function $\phi: Y \rightarrow[-\infty, \infty)$ is pluriharmonic if and only if $\phi$ is weakly pluriharmonic and regularized in $Y$.

\section{Applications}

As a consequence of Theorem 3.1, subharmonicity and plurisubharmonicity are closed under a basic sequential limiting process (Lemma 6.1). By allowing to decrease the values of a subharmonic (respectively, plurisubharmonic) function on a null set, the failure of the preservation of subharmonicity (respectively, plurisubharmonicity) under more general limit operations can be remedied. A mapping $\phi: Y \rightarrow[-\infty, \infty)$ is called presubharmonic $(\phi \in \mathcal{S H}[Y])$ (respectively, preplurisubharmonic, $(\phi \in \mathcal{P S H}[Y]))$ if $\phi$ admits a subharmonic (respectively, plurisubharmonic) majorant $\hat{\phi}: Y \rightarrow[-\infty, \infty)$ such that $\phi=\hat{\phi}$ almost everywhere.

Lemma 5.1. (Compare [9, Theorem L8]) A mapping $\phi: Y \rightarrow[-\infty, \infty)$ is presubharmonic (respectively, preplurisubharmonic) if and only if the upper envelop

$$
\phi^{[*]}(z):=\max \left(\phi(z), \phi^{(*)}(z)\right), \quad \forall z \in Y,
$$

is subharmonic (respectively, plurisubharmonic), with $\phi^{[*]}=\phi$ almost everywhere, in $Y$; hence $\phi^{[*]}$ is the least such majorant of $\phi$.

Proof. The sufficiency part of the lemma is trivial. Suppose that $\phi$ admits a subharmonic majorant $\hat{\phi}$ which agrees with $\phi$ almost everywhere. Then the same argument as in the proof of $[9$, Theorem L8] shows that $\phi(z) \leq \phi^{[*]}(z) \leq \hat{\phi}^{[*]}(z)=\hat{\phi}(z)$ for all $z \in Y$ and hence $\phi(z)=\phi^{[*]}(z)$ almost everywhere. On the other hand, if $\phi^{[*]}(a)<\hat{\phi}(a)$ at some $a \in Y$, then by the definition (5.1), there are constants $\varepsilon>0, \delta>0$, such that $\sup _{z \in \Delta_{a}(\varepsilon)} \phi(z)=\hat{\phi}(a)-\varepsilon$. But since $\hat{\phi}$ is subharmonic and $\hat{\phi}=\phi$ almost everywhere in $Y$, it follows from the regularity of $\hat{\phi}$ (Proposition 3.2) that, for some standard domain $\Delta$ at $a$,

$$
\left.\nu_{p_{\Delta}}(a) \hat{\phi}(a)=\lim _{r \rightarrow 0}\langle\phi\rfloor \Delta\right\rangle_{a, r} \leq \nu_{p_{\Delta}}(a)(\hat{\phi}(a)-\varepsilon),
$$

a contradiction, thus proving the desired claim. The remaining case of preplurisubharmonicity is entirely similar.

Thanks to Corollary 4.1 and Lemma 5.1, the preservation of preplurisubharmonicity on a complex space can be assured in reference to: (1) the pointwise limit of a monotonically decreasing sequence in 
$\mathcal{P S H}[Y] ;(2)$ the supremum and lim sup of a sequence in $\mathcal{P S H}[Y]$ that is locally uniformly bounded from above (as in [9, Theorems L9]); and, for a normal space $Y$, (3) the supremum of a family $\left\{\phi_{t}\right\}_{t \in T} \subset \mathcal{P S H}[Y]$ that is locally uniformly bounded from above (as in [9, Theorems L10]), and (4) the operation of forming $\psi_{t_{0}}:=\limsup _{t \rightarrow t_{0}} \phi_{t}$, for $\left\{\phi_{t}\right\}_{t \in T} \subset \mathcal{P S H}[Y]$ that is locally uniformly bounded from above (where $t_{0} \in T$ and $T$ is an open (or closed) subset of $\mathbb{R} \cup\{\infty\}$ or $\mathbb{C}^{m}$ ). Similar assertions hold on a general space for presubharmonic functions. An application of such preservation properties is given by the following generalization of the Hartogs' lemma:

Corollary 5.1. (Hartogs' lemma for presubharmonic functions) Assume that $\left\{\phi_{t}\right\}_{t>0}$ is a family in $\mathcal{S H}[Y]$ that is locally uniformly bounded from above. Then (1) the function $G:=\limsup _{t \rightarrow \infty} \phi_{t}$ is presubharmonic in $Y$; (2) if for some $g \in C^{0}(Y), G \leq g$ in $Y$, then for every compact set $K \subset Y$ and every positive $\varepsilon$, there is a positive number $N$ such that $\sup _{K} \phi_{t}<g+\varepsilon$ for all $t \geq N$.

Proof. Let $F_{t}:=\sup _{s \geq t} \phi_{s}$. Then $G=\lim _{t \rightarrow \infty} F_{t}$. By the preceding remark, both $F_{t}$ and $G$ are presubharmonic in $Y$, (alternatively, this can be proved using Theorem 3.1 and Lemma 6.2). Since $\left\{F_{t}\right\}$ is a decreasing sequence, so is also the sequence $\left\{F_{t}^{[*]}\right\}$, hence the limit function $\lim _{t \rightarrow \infty} F_{t}^{[*]}$ is subharmonic. It follows from Lemma 5.1 that $G^{[*]}$ is equal to $\lim _{t \rightarrow \infty} F_{t}^{[*]}$ outside a set of measure zero, and hence by Proposition 3.2, everywhere in $Y$. The second assertion follows by showing that the nested family $E_{t}:=\left\{z \in K \mid F_{t}^{[*]}(z)-g(z) \geq \varepsilon\right\}$, for $t>0$, has an empty intersection (by the same argument as in $[12$, p. 93]).

In a similar vein it is of interest to see if the theorem of Lelong-Norguet-Bremermann [13, p. 54] on the representation of plurisubharmonic functions remains valid for a $P$-convex domain $D$ in a general complex space, where $P=P S H(D)$, that is, whether each $\psi \in P S H(D)$ admits an representation

$$
\psi=\left(\limsup _{k \rightarrow \infty} \frac{1}{k} \log \left\|f_{k}\right\|\right)^{[*]},
$$

for some sequence of holomorphic functions $f_{k}$ in $D$.

Let $p: Y \rightarrow \Omega$ be a Riemann semicovering and $G \Subset Y$ a weak Stokes domain ([24, p. 568]). The (real) energy of a function $\tau \in C^{1}(\bar{G})$ is defined by

$$
\mathbb{E}_{G}(\tau):=\int_{G} d \tau \wedge d^{c} \bar{\tau} \wedge v_{p}^{m-1}
$$

([25, § 3]). If $\tau \in C^{1,1}(\bar{G})([24$, p. 562$])$ is real-valued, then its energy is given by

$$
\mathbb{E}_{G}(\tau)=\int_{d G} \tau d^{c} \tau \wedge v_{p}^{m-1}-\int_{G} \tau d d^{c} \tau \wedge v_{p}^{m-1} .
$$

Suppose now that $p: Y \rightarrow \Omega$ is an unramified Riemann covering. By the expression [24, (5.7)] for the normal derivatives one has

$$
\int_{d G} \tau, d^{c} \tau \wedge v_{p}^{m-1}=(-1)^{\frac{m(m-1)}{2}} \frac{1}{2\|\mathbb{S}\|} \int_{d G} \tau \partial_{\nu} \tau d \sigma_{d G}
$$


Also, since the energy $\mathbb{E}_{G}(\tau)$ is never negative [24, (3.7)], the expression (5.2) (applied to the pseudoannular region $G=U_{a}(r) \backslash \bar{U}_{a}(s)$, where $\left.0<s<r<r_{0}\right)$ and the identity (5.6) of [24] imply that the spherical mean $\left[\tau \mathfrak{R}_{p, a}(\tau)\right]_{a, r}$ of a subharmonic $C^{2}$-function $\tau: Y \rightarrow[0, \infty)$ is nonnegative and increasing in $r$ (for small $r>0$ ).

If $\phi=\left(\phi_{1}, \cdots, \phi_{N}\right): Y \rightarrow \mathbb{C}^{N}$, set $\tau_{\rho}:=\|\phi\|^{2 \rho}$ for each positive constant $\rho$. A mapping $\phi: Y \rightarrow \mathbb{C}^{N}$ is called subharmonic, respectively, semiharmonic, if so are the real and imaginary parts of each component of $\phi$ (regarded as an $\mathbb{R}$-valued function); $\phi$ is called weakly real-analytic if each component $\phi_{j}$ is continuous in $Y$ and real-analytic in $Y_{\text {reg. }}$ Kellog ([11, Theorem IV, p. 213]) showed that a real-valued harmonic function on a closed, regular plane region is nonconstant unless it has vanishing normal derivative at every boundary point. The relations (5.2) and (5.3) lead to a generalization of this result to subharmonic mappings:

Proposition 5.1. (1) Assume that $G \Subset Y$ is a Stokes domain and $\phi: \bar{G} \rightarrow \mathbb{C}^{N}$, with components $\phi_{j} \in C^{1,1}(\bar{G}) \cap C^{2}(G)$, is subharmonic in $G$. If for some constant $\rho \geq \frac{1}{2}$,

$$
\int_{d G} \tau_{\rho} d^{c} \tau_{\rho} \wedge v_{p}^{m-1}=0
$$

then $\phi=$ constant in $\bar{G}$. (2) Assume that $Y$ is a connected complex space and $\phi: Y \rightarrow \mathbb{C}^{N}$ a weakly real-analytic subharmonic map. Then $\phi$ is a constant if and only if there exist a point $a \in Y_{\text {reg }}$ and $\rho \geq \frac{1}{2}$ such that the function $\left[\tau_{\rho} \mathfrak{R}_{p, a}\left(\tau_{\rho}\right)\right]_{a, r}$ is monotonically decreasing in $r$ (for small $r>0$ ) relative to an unramified Riemann covering $p$ at a.

Proof. By Example 6.1 of the Appendix and Lemma 3.2, the function $\tau_{\rho}=\|\phi\|^{2 \rho}$ is subharmonic in $G$ for all $\rho \geq \frac{1}{2}$. (1) Observe that

$$
d d^{c}\left\|\phi_{j}\right\|^{2}=\bar{\phi}_{j} d d^{c} \phi_{j}+\phi_{j} d d^{c} \bar{\phi}_{j}+d \phi_{j} \wedge d^{c} \bar{\phi}_{j}-d^{c} \phi_{j} \wedge d \bar{\phi}_{j}
$$

almost everywhere in $G$. Writing $\phi_{j}=u_{j}+i v_{j}$ (in terms of its real and imaginary parts), one has $\|\phi\|^{2}=\sum_{j=1}^{N}\left(\left\|u_{j}\right\|^{2}+\left\|v_{j}\right\|^{2}\right)$. Thus the mapping

$$
\tilde{\phi}:=\left(\left|u_{1}\right|,\left|v_{1}\right|, \cdots,\left|u_{N}\right|,\left|v_{N}\right|\right): \bar{G} \rightarrow \mathbb{R}^{2 N}
$$

is continuous in $\bar{G}$ and subharmonic in $G$ with $\|\tilde{\phi}\|^{2}=\|\phi\|^{2}$. Therefore it can be assumed without loss of generality that each $\phi_{j}$ is real-valued. Then the expression (5.5) implies that

$$
d d^{c} \tau_{1} \wedge v_{p}^{m-1} \geq 2 \sum_{j=1}^{N}\left(d u_{j} \wedge d^{c} u_{j}+d v_{j} \wedge d^{c} v_{j}\right) \wedge v_{p}^{m-1} \geq 0
$$

almost everywhere in $G$. To prove the assertion (1), observe that by the condition (5.4) and Proposition 3.4, the energy (5.2) (for $\tau_{\rho}$ ) is nonpositive. Since the integral $\mathbb{E}_{G}\left(\tau_{\rho}\right)$ is always nonnegative, the norm of the gradient, $\left\|\nabla \tau_{\rho}\right\|$, must vanish locally almost everywhere in $G$. Consequently $\tau_{\rho}$, hence also $\tau_{1}$, is constant in $G$. Hence the relation (5.6) implies that $\phi=$ constant in $G$. For the assertion (2), assume that relative to an unramified Riemann covering $p$ at a point $a \in Y_{\text {reg }}$, the function $\left[\tau_{\rho} \Re_{p, a}\left(\tau_{\rho}\right)\right]_{a, r}$ is decreasing in $r$ for some $\rho \geq \frac{1}{2}$. Then by the expression $(5.2)$, the energy $\mathbb{E}_{D}\left(\tau_{\rho}\right)$ 
for the pseudoannular region $G:=U_{a}(r) \backslash \bar{U}_{a}(s), 0<s<r<r_{0}$, is nonpositive. It follows that $\left\|\nabla \tau_{\rho}\right\|^{2}=0$ in $G$. Therefore $\tau_{\rho}$ is constant in every component of $G \backslash Z$, where $Z$ is the zero set of $f=\|\phi\|^{2}$. Since each component $\phi_{j}$ of $\phi$ is real analytic in $Y$ off a thin analytic subset, the same is true for the function $f$; hence (choosing $a$ to be a point where $f$ is real analytic and $r_{0}$ sufficiently small) the closure of every component of $G \backslash Z$ must contain a ([16, Lemma 1, p. 96]). This implies that $\tau_{\rho}$ is constant in $G$; in particular, so is $\tau_{1}$. It follows as above from the relation (5.6) that $\phi=$ constant in $G$. Hence by the identity theorem for real-analytic functions, $\phi=$ constant in $Y$.

Corollary 5.2. Let $Y$ be a connected complex space and $\phi=\left(\phi_{1}, \cdots, \phi_{N}\right): Y \rightarrow \mathbb{C}^{N}$ a weakly real-analytic and subharmonic map. If $\|\phi\|$ attains a local maximum at some point of $Y$, then $\phi=$ constant.

Proof. Observe that the function $\tau_{1}=\|\phi\|^{2}: Y \rightarrow \mathbb{R}$ is solidly submedian in $Y$. Assume that $\|\phi\|$ attains a local maximum at a point $z_{0} \in Y$. Hence Proposition 3.1 (applied to a Riemann covering $p_{U}$ of a small open, connected neighborhood $U$ of $z_{0}$ ) implies that $\tau_{1}$ is constant in $U$. Thus $\tau_{1}$ has vanishing mean spherical radial derivative at some point of $U$, and by Proposition 5.1-(2), $\phi=$ constant in $U$. Since each $\phi_{j}$ is real analytic in $Y_{\text {reg }}, \phi_{j}=$ constant in $Y_{\text {reg }}$ by the identity theorem for real-analytic functions, whence the conclusion follows.

By means of the solid submean-value property of subharmonic functions on a general space, two results of Bochner and Montgomery on matrix-valued mappings [2, Theorems 10 and 11, p. 155] can be extended:

Proposition 5.2. Assume that $Y$ is a connected complex space. (1) If $\phi=\left(\phi_{1}, \cdots, \phi_{k N}\right): Y \rightarrow \mathbb{R}^{k N}$ is continuous, subharmonic such that the image of each map $g_{j}:=\left(\phi_{(j-1) N+1}, \cdots, \phi_{j N}\right): Y \rightarrow \mathbb{R}^{N}, 1 \leq$ $j \leq k$, is contained in the unit sphere in $\mathbb{R}^{N}$, then $\phi=$ constant. (2) Every continuous mapping $\phi: Y \rightarrow \mathrm{U}(n)$ (a unitary group) with semiharmonic components is a constant.

\section{Appendix}

Lemma 6.1. If $\left\{\phi_{n}\right\}$ is a monotonically decreasing sequence of subharmonic (respectively, plurisubharmonic) functions in a complex space $Y$, then the limit function $\lim \phi_{n}$ is subharmonic (respectively, plurisubharmonic) in $Y$.

Lemma 6.2. Assume that (i) $Y$ is locally irreducible; (ii) $\psi: Y \rightarrow[-\infty, \infty$ ) is locally integrable and locally bounded from above; and (iii) $\psi$ is solidly submedian with respect to every pseudoball in $Y$. Then $\psi$ is presubharmonic in $Y$.

Proof. Since $\psi$ is locally bounded from above, it can be proved in the same way as in [9, Lemma L6-(c)] that $\psi^{[*]}$ is upper semicontinuous in $Y$. Let $\Delta$ be a pseudoball at at point $z \in Y$. Then either $\psi^{[*]}(z)=\psi(z)$, hence the solid submean-value inequality holds at $z$, or there exists a sequence $\left\{\zeta_{n}\right\}$ in $\Delta \backslash\{z\}$ tendng to $z$ such that $\psi^{[*]}(z)=\lim _{n \rightarrow \infty} \psi\left(\zeta_{n}\right)$; in the latter case one has

$$
\left.\left.\left.\psi^{[*]}(z) \leq \liminf _{n \rightarrow \infty}\langle\psi\rfloor \Delta\right\rangle_{\zeta_{n}, r} \leq \liminf _{n \rightarrow \infty}\left\langle\psi^{[*]}\right\rfloor \Delta\right\rangle_{\zeta_{n}, r}=\left\langle\psi^{[*]}\right\rfloor \Delta\right\rangle_{z, r},
$$


for sufficiently small $r>0$. Thus $\psi^{[*]}$ is subharmonic in $Y$ by Theorem 3.1. Also,

$$
\left.\left.\psi^{[*]}(z) \leq \liminf _{n \rightarrow \infty}\langle\psi\rfloor \Delta\right\rangle_{\zeta_{n}, r}=\langle\psi\rfloor \Delta\right\rangle_{z, r} .
$$

It follows then from the regularity of $\psi^{[*]}$ that

$$
\left.\left.\lim _{r \rightarrow 0}\left\langle\psi^{[*]}\right\rfloor \Delta\right\rangle_{z, r}=\lim _{r \rightarrow 0}\langle\psi\rfloor \Delta\right\rangle_{z, r} .
$$

Consequently $\psi^{[*]}=\psi$ almost everywhere in $Y$.

Lemma 6.3. (Compare $[17,3.13])$ Let $g: I \rightarrow \mathbb{R}$ be an increasing function on a finite (or infinite) interval $I \subseteq[-\infty,-\infty)$ such that $g\rfloor I \cap \mathbb{R}$ is convex. Assume that $\phi \in S H(Y)$ (respectively, $\phi \in$ $P S H(Y)$ ) with $\phi(Y) \subset I$. Then $g(\phi) \in S H(Y)$ (respectively, $g(\phi) \in P S H(Y)$ ).

Proof. Suppose that $\phi \in S H(Y)$. Let $\Delta$ be a pseudoball at a point $a \in Y$ of radius $r_{0}$. By Theorem 3.1, one has $\phi(a) \leq\langle\phi\rfloor \Delta\rangle_{a, r}$ for every $r \in\left(0, r_{0}\right)$. Note that, since $g(\phi)=g^{+}(\phi)-g^{-}(\phi)$, the function $g(\phi)$ is locally integrable on $Y$. Using the supporting line argument of [19, p. 115], it can be shown that the Jensen's inequality for convex functions holds for the pair $(g, \phi)$ :

$$
\left.\left.g(\langle\phi\rfloor \Delta\rangle_{a, r}\right) \leq\langle g \circ \phi\rfloor \Delta\right\rangle_{a, r}, \quad \forall r \in\left(0, r_{0}\right) .
$$

Therefore, by Theorem 3.1, the composition $g \circ \phi$ is subharmonic in $Y$. The corresponding assertion for $\phi \in \operatorname{PSH}(Y)$ follows then from the definition of plurisubharmonicity.

Example 6.1. Assume that $\phi=\left(\phi_{1}, \cdots, \phi_{N}\right): Y \rightarrow \mathbb{C}^{N}$ is a subharmonic map with components $\phi_{j} \in C^{2}(Y \backslash A)$ for some thin analytic subset $A$ of $Y$. Then for all constants $c, \rho$ with $c>1, c \rho \geq 1$, the function $\left(\lfloor\phi\rfloor^{\hat{c}}\right)^{\rho} \in S H(Y)$ (where $\hat{c}=(c, \cdots, c)$ ).

Proof. It is a consequence of $[17, \mathbf{3 . 2 3}$, p. 19], Proposition 3.4 and Lemma 3.2 that the function $g:=\left(\lfloor\phi\rfloor^{\hat{c}}\right)^{1 / c}$ is subharmonic in $Y$, hence so is $g^{c \rho}=\left(\lfloor\phi\rfloor^{\hat{c}}\right)^{\rho}$ by Lemma 6.3, provided $c \rho \geq 1$.

Lemma 6.4. (Compare $[17,2.13])$ Assume that $\phi: Y \rightarrow[0, \infty)$ is upper semicontinuous and $\phi \not \equiv 0$. Then $\phi$ is logarithmically subharmonic in $Y$ if and only if $\phi e^{h} \in S H(D)$ for every subdomain $D \Subset Y$ and every continuous function $h: \bar{D} \rightarrow \mathbb{R}$ which is semiharmonic in $D$. In particular, if $\phi$ is logarithmically subharmonic in $Y$, then $\phi \in S H(Y)$. The converse is false.

Proof. By virtue of Proposition 3.1 and Lemmas 3.2 and 6.3, the desired conclusion follows from the same argument as in $[17,2.13]$.

Example 6.2. If $\phi_{k}: Y \rightarrow[0, \infty)$ is upper semicontinuous and logarithmically subharmonic for $1 \leq k \leq l$ then so are $\sum_{k=1}^{l} \phi_{k}$ and $\max _{1 \leq k \leq l} \phi_{k}$.

Example 6.3. (Compare [4, p. 119]) Let $F_{j}=\left(f_{j 1}, \cdots, f_{j N}\right): Y \rightarrow \mathbb{C}^{N}$ be a holomorphic map for each $1 \leq j \leq s$. Then for all constants $\rho>0$ and $c_{(j)}=\left(c_{j 1}, \cdots, c_{j N}\right)$ with $c_{j k}>0$, the functions $\left(\left\lfloor F_{j}\right\rfloor^{c_{(j)}}\right)^{\rho}$ and $\max _{1 \leq j \leq s}\left\lfloor F_{j}\right\rfloor^{c_{(j)}}$ are logarithmically plurisubharmonic in $Y$. 
Remark 6.1. If $\phi: Y \rightarrow[0, \infty)$ is logarithmically plurisubharmonic, it can be shown as in Lemma 6.4 that, for every subdomain $D \Subset Y$ and every continuous function $h: \bar{D} \rightarrow \mathbb{R}$ which is semiharmonic in $D$, one has $\phi e^{h} \in P S H(D)$ (in particular, $\phi \in P S H(Y)$ ). In the converse direction, using Theorem 4.1 the following Lemma can be proved (as in [12, Theorem 2.6.1]):

Lemma 6.5. (Compare also [3, Proposition VI.4.9] and, respectively, $[17,3.12]$ ) Let $(X, p)$ be a normal semi-Riemann domain. Assume that $\phi: X \rightarrow[0, \infty)$ is upper semicontinuous and locally $\phi \not \equiv$ 0 . Then $\phi$ is logarithmically plurisubharmonic (respectively, logarithmically subharmonic) in $X$ if and only if $\phi\left|e^{\sum c_{k} p_{k}}\right| \in P S H(X)$ (respectively, $\left.\phi\left|e^{\sum c_{k} p_{k}}\right| \in S H(X)\right)$ for all constants $\left(c_{1}, \cdots, c_{m}\right) \in \mathbb{C}^{m}$.

Received: January 2009. Revised: May 2009.

\section{References}

[1] H. Behnke and H. Grauert, Analysis in non-compact complex spaces, Analytic functions (H. Behnke and H. Grauert,ed.), Princeton Univ. Press, Princeton, New Jersey, 1960.

[2] S. Bochner and W. Martin, Several complex variables, Princeton University Press, Princeton, New Jersey, 1948.

[3] D'Angelo, John P., Inequalities from complex analysis, The Carus Math. Mono. Number 28,Math. Assoc. Amer., Washington DC, 2002.

[4] Demailly, Jean-Pierre, Courants positifs et théorie de l'intersection, Gaz. des Math. No. 53 (1992), 131-159.

[5] J. Forness and R. Narasimhan, The Levi problem on complex spaces with singularities, Math. Ann. 248 (1980), 47-72.

[6] P. Freitas and J. P. Matos, Characterization of harmonic and subharmonicfunctions via meanvalue properties, arXiv:math/010607801v1 (2001), 1-10.

[7] D. Gilbarg and N. S. Trudinger, Elliptic partial differential equations of second order, Springer-Verlag, Berlin-Heidelberg-New York, 1977.

[8] H. Grauert and R. Remmert, Plurisubharmonischen Funktionen in komplexen Räume, Math. Zeit. 65 (1956), 175-194.

[9] R. C. Gunning, Introduction to holomorphic functions of several variables, vol. I: function theory, Wadsworth, Belmont, California, 1990.

[10] L. Hörmander, An introduction to complex analysis in several variables, D. Van Nostrand, Princeton, New Jersey, 1966.

[11] W. Kellog, Foundations of potential theory, Dover, New York, 1953.

[12] P. Lelong, Fonctionnelles analytiques et fonctions entièrs (n variables), L'Univ. de Montréal, 1968. 
[13] P. Lelong, Plurisubharmonic functions and positive differential forms, Gordon and Breach, New York, London, Paris, 1969.

[14] E. H. Lieb And M. Loss, Analysis,Graduate Studies in Math. Vol. 14, Amer.Math. Soc., 1996.

[15] R. Narasimhan, The Levi problem for complex spaces, Math. Ann. 142 (1961), 355-362.

[16] R. Narasimhan, Introduction to the theory of analytic spaces, Lecture Notes in Math. 25, Springer, Berlin-Heidelberg-New York, 1966.

[17] T. RADÓ, Subharmonic functions, Ergebnisse der Mathematik und ihrer Grenzgebiete, Springer, Berlin, 1937.

[18] T. Radó And P. Reichelderfer, Continuous transformations in analysis, Springer-Verlag, Berlin-Heidelberg, 1955.

[19] H. L. Royden, Real analysis, third edition, Macmillan, New York, London, 1988.

[20] B. V. Shabat, Introduction to complex analysis Part II, Amer. Math. Soc., 1992.

[21] W. Stoll, The multiplicity of aholomorphic map, Invent. Math. 2,(1966), 15-58.

[22] W. Stoll, Value distribution on parabolicspaces, Lecture Notes in Math. 600, Springer,BerlinHeidelberg-New York, 1977.

[23] C. Tung, The first main theoremof value distribution on complex spaces, Memoiredell'Accademia Nazionale dei Lincei, Serie VIII,Vol. XV, (1979), Sez.1, 91-263.

[24] C. Tung, Semi-harmonicity, integral means and Euler type vector fields, Advances in Applied Clifford Algebras, 17 (2007), 555-573.

[25] C. Tung, Integral products, Bochner-Martinelli transforms and applications, Taiwanese Journal of Mathematics, 13 no. 5 (2009), 1583-1608.

[26] V. Vladmirov, Methods of the theory of functions of many complex variables, The MIT Press, Cambridge and London, 1966. 